\title{
LA NORMALIZACIÓN DE LOS DELITOS SEXUALES EN COSTA RICA: UNA CUANTIFICACIÓN
}

\section{NORMALIZATION OF SEXUAL CRIMES IN COSTA RICA: A QUANTIFICATION}

\author{
Laura C. Blanco ${ }^{1}$ \\ Sergio E. Rojas Peralta ${ }^{2}$
}

Recibido: 10/10/2019

Aprobado: 22/07/2021

\section{RESUMEN}

\begin{abstract}
A partir de la Encuesta Nacional de Salud Sexual y Reproductiva (2010 y 2015), se estima que más de una quinta parte de la población mayor de 15 años reporta experiencias que las colocaría en la posición de víctima de relaciones sexuales no consentidas, según los criterios establecidos por la Ley 9406. No obstante, únicamente 10\% de estas personas reporta haber sido víctima de delitos sexuales. Esta ausencia de percepción de algún delito pone en evidencia patrones culturales más permisivos, en cuanto al consentimiento sexual. Este tema es discutido a través de un análisis de la normativa a lo largo de la historia. Posteriormente, se utiliza el modelo de capital humano para estimar regresiones logit sobre la probabilidad de ser víctima de este tipo de delitos sexuales. Se encuentra que una mejor educación y una comunicación satisfactoria con progenitores y educadores reducen la probabilidad de ser víctima de dichos delitos.
\end{abstract}

PALABRAS CLAVE: : COMPORTAMIENTO ILEGAL, DELITOS SEXUALES, ABUSO, RELACIÓN IMPROPIA, CONSENTIMIENTO.

CLASIFICACIÓN JEL: : K400, K420

\section{ABSTRACT}

The National Survey on Sexual and Reproductive Health (2010 y 2015) is used to estimate that over a fifth of the population age 15 and over reports experiences that would place them as victims of sexual relations without consent according to the criteria established in the Law 9406. However, only $10 \%$ of these people report having been a victim of sexual crimes. This lack of perception of any crime points to the existence of more permissive cultural patterns regarding sexual consent. This is discussed through an analysis of the legislation throughout history. Later, the human capital model is used to estimate logit regressions on

1 Universidad de Costa Rica, Escuela de Economía; Código postal 11501-2060; San José, Costa Rica; lauracristina.blanco@ucr.ac.cr

2 Universidad de Costa Rica, Escuela de Filosofía; Código postal 11501-2060; San José, Costa Rica; sergio.rojas@ucr.ac.cr 
the probability of being a victim of these sex crimes. Results show that better education and a satisfactory communication with parents and teachers reduce the probability of being a victim of such crimes.

KEYWORDS: ILLEGAL BEHAVIOR, SEXUAL CRIMES, ABUSE, IMPROPER RELATION, CONSENT. JEL CLASSIFICATION: K400, K420

\section{INTRODUCCIÓN}

El 13 de enero de 2017, entró a regir la Ley 9406 Fortalecimiento de la protección legal de las niñas y las adolescentes mujeres ante situaciones de violencia de género asociadas a relaciones abusivas, la cual establece las penas por mantener relaciones sexuales con personas menores de edad. A partir de los criterios de rangos de edad establecidos en dicha ley, se realizó su cuantificación utilizando la Encuesta Nacional de Salud Sexual y Reproductiva para los años 2010 y 2015, con el fin de conocer el porcentaje de la población que podría verse afectada por esta ley. Se encuentra que al menos una quinta parte de la población mayor de 15 años ha sido víctima de delitos sexuales, pero únicamente $10 \%$ de ella reporta haber sido víctima de abuso. Además de aportar una medición del nivel de abuso reflejado en la Encuesta, este artículo contribuye a la discusión sobre los delitos sexuales al utilizar un abordaje interdisciplinario y un análisis correlacional para identificar aquellas variables asociadas a la probabilidad de ser víctima de delitos sexuales, con el fin de empezar a dilucidar un perfil de la población que podría estar más expuesta a este tipo de situaciones. Este es el primer trabajo en realizar un análisis interdisciplinario de este tipo, ya que la Encuesta lo permite. Es evidente que la Ley 9406 apunta a modificar las prácticas sexuales y la manera de concebir las relaciones abusivas cuantificadas en la Encuesta. Esta, contrastada con la historia legal, permite ver cuál es la percepción del delito sexual que tiene la población, al margen de la noción o percepción que tiene el legislador. Evidentemente, cualquier diferencia entre una y otra percepción indicaría un problema de educación, cultura o sensibilización sobre el asunto, que es precisamente la inconsistencia que se observa en la encuesta.

En la sección segunda se presenta una discusión sobre la edad de consentimiento, la evolución del concepto a lo largo de la historia, las problemáticas filosóficas y de derechos humanos que surgen alrededor de ella, las reacciones contestatarias y por qué en este artículo se ha optado por abordar este tema desde los criterios legales y no desde las posiciones críticas que parecen oponerse a la existencia de la regulación por edad. La sección tercera resume la evolución de la edad de consentimiento en Costa Rica a partir de una revisión de los códigos penales. La sección cuarta presenta la metodología utilizada para construir la tasa de delitos sexuales, así como la base de datos utilizada. Por su parte, la sección quinta presenta los resultados de un análisis de regresión logit para identificar aquellas variables asociadas a los delitos sexuales en la muestra y la sección sexta concluye. Esto nos permite abordar el problema de la normalización de los delitos sexuales desde un análisis multidisciplinario, al integrar un análisis desde la filosofía, el derecho, el uso de métodos cuantitativos y conceptos económicos, para comprender la magnitud de los delitos sexuales en Costa Rica, así como la falta de correspondencia entre estos delitos y el reconocimiento de su existencia por parte de la población, quien parece normalizarlos y aceptarlos en su diario vivir.

\section{LOS DELITOS SEXUALES Y LA EDAD DE CONSENTIMIENTO}

Las leyes relacionadas con la edad de consentimiento son aquellas que criminalizan las relaciones sexuales con personas menores de edad, pues dichas relaciones constituyen violaciones sexuales de tipo estatutario. El argumento subyacente consiste en que una persona menor a la edad establecida estatutariamente no es capaz de comprender todas las implicaciones que 
acarrea un acto sexual $y$, por tanto, es incapaz de tomar una decisión libre y razonada (Frank, Camp \& Boutcher, 2010, p. 875). Esto la hace, a su vez, más vulnerable a la manipulación por parte de personas adultas (Bierie \& Budd, 2018), exponiéndola así a peligros sobre su integridad e individualidad. Quien mantiene relaciones sexuales con una persona menor de edad estaría, entonces, abusando de ella, al aprovecharse de su incapacidad para consentir, aun cuando no medie un acto violento de por medio. Actualmente, estas leyes se promueven como un mecanismo para la protección de las personas menores de edad contra la explotación sexual (Bierie \& Budd, 2018; Unicef, 2018), particularmente las mujeres, de forma que se enmarcan en un discurso universal de derechos humanos. Sin embargo, este enfoque es reciente y no corresponde a la evolución histórica en Occidente de la edad de consentimiento, en general.

Inicialmente, la legislación relativa a la edad de consentimiento se refería a crímenes contra la propiedad, en tanto pretendía salvaguardar la virginidad de las mujeres. Esto protegía o aseguraba la validez de los contratos matrimoniales negociados entre los padres y esposos de dichas mujeres (Cocca, 2015), i.e., la virginidad de la hija era propiedad del padre y atentar contra ella implicaba una pérdida de valor sobre el bien de este. La edad de consentimiento aparece primero como una competencia legal en Grecia y rondaba los 12 años, tanto para mujeres como para hombres, haciéndola coincidir con la pubertad (Bullough, 2005). Esta edad de consentimiento difería de la edad de matrimonio para los hombres, quienes se iniciaban sexualmente en su pubertad con otros hombres, pero contraían matrimonio alrededor de los 30 años con mujeres, usualmente púberes (Bullough, 2005). Lo anterior le aseguraba al hombre el acceso al cuerpo de una mujer virgen y, por tanto, certeza sobre su descendencia.

Similarmente, la edad de consentimiento en Roma se estableció en 12 años para las mujeres y 14, para los hombres, aunque se estima que rondaba, en promedio, los 14 años en ambos casos. Hacia el final del imperio, la edad de consentimiento mínima se estableció en siete años, ya que se consideraba que esta era la edad en que las personas eran capaces de entender a qué estaban consintiendo (Bullough, 2005). Este criterio se extiende a la Europa de la Edad Media a través del Corpus Iuris Civilis, el código civil de Justiniano en el siglo VI (Bullough, 2005). En el siglo XIII, aparece en Inglaterra la primera legislación de una nación europea sobre el tema (Cocca, 2015), cuando el Estatuto de Westminster de 1275 establece explícitamente los 14 años como edad de consentimiento (Edward I, 1275). Posteriormente, los criterios del código de Justiniano se consolidan en el Concilio de Trento en 1563 (Bullough, 2005). A pesar de este límite de edad, era usual que los progenitores acordaran el matrimonio a edades inferiores a los 12 años, pues podían ejercer su patria potestad para consentir en lugar de la persona. Aunque para que el matrimonio tuviese validez, las personas contrayentes debían consentir y estar en capacidad física para consumarlo.

Estas normas estuvieron vigentes hasta la modernidad, cuando fueron momentáneamente sustituidas por el Código Napoleónico de 1804, el cual establecía los criterios de edad y consentimiento para establecer la edad mínima de matrimonio, 18 años para el hombre, 15 para la mujer (art.144)2. Esto desencadenó una serie de reformas en los diferentes países europeos y sus colonias, quienes elevaron la edad de consentimiento a un rango entre los 12 y 16 años (Bullough, 2005). En esta etapa, la regulación sobre la edad de consentimiento se refería a crímenes tanto contra la propiedad como contra el honor (Cocca, 2015). En los países protestantes, especialmente aquellos vinculados a los movimientos evangélicos, como Estados Unidos, el ascenso del puritanismo en la primera mitad del siglo XIX promovió un discurso que presentaba a la prostitución como la principal fuente de la corrupción social y aspiraba a imponer la pureza social

2 Dichas edades diferían, además, de la edad general de minoría (21 años, según su art.488), constituida la edad para el matrimonio como una excepción a dicha regla. 
y preservar la virtud. En este país, la edad de consentimiento se elevó de 10 años a entre 16 y 18 en los diferentes estados (Ehrlich, 2006). Paralelo a este movimiento puritano, se halla también las consignas del primer movimiento feminista durante la época victoriana: las sufragistas, tanto en Estados Unidos como Inglaterra, denunciaban la existencia de un doble estándar que permitía, por un lado, la depravación masculina y castigaba, por otro, a las mujeres, por lo que exigían iguales estándares morales a mujeres y hombres, tomando el de la mujer como el estándar superior (Cocca, 2015; Ehrlich, 2006), lo que imponía un precepto de virtud en el ámbito sexual y generaba presión para elevar la edad de consentimiento.

A inicios del siglo XX, se empezó a reconocer que algunas veces las personas actúan por deseo propio, pero lejos de afirmar la libertad individual, este reconocimiento del deseo resultó problemático en el marco de los valores victorianos, por lo que se reprimió a través de la figura de la delincuente sexual (Ehrlich, 2006). De esta forma, las mujeres, y las prostitutas en particular, pasan de ser víctimas de la corrupción masculina a ser delincuentes y, por tanto, responsables de los crímenes sexuales y el mal comportamiento de los hombres (Ehrlich, 2006). Esto introduce un trato diferenciado entre mujeres $y$ hombres en los sistemas penales que buscaba castigar la promiscuidad de las mujeres, a la vez que dejaba impune a los hombres, lo cual representó, indudablemente, un retroceso respecto a las demandas de igualdad externadas por el movimiento feminista hacia finales del siglo XIX.

La reclasificación de los crímenes contra el honor y la moralidad como crímenes contra la libertad individual o integridad física no se dio hasta después de la Segunda Guerra Mundial (Frank et al., 2010). Esta reclasificación obedeció a un reordenamiento de las instituciones sociales, las cuales pasaron de enfocarse en los grupos colectivos (tales como la familia o la nación), a las personas individualmente (Frank et al., 2010). Dicha reconstitución social se definió y dictó, además, desde los organismos internacionales, los cuales aspiraban a establecer normas fundamentadas en un carácter científico, profesional y universal. Las instituciones nacionales empezaron así a adoptar estas normas negociadas internacionalmente, de forma que se produjo una tendencia hacia la estandarización del marco legal entre los países. Consecuentemente, entre 1945 y 2005 se observó una serie de reformas sobre la penalización de las ofensas sexuales en todo el mundo, incluidas la violación y el abuso de menores:

la violación sexual se penaliza precisamente porque viola la regla sobre el consentimiento, mientras que el abuso sexual infantil explota la incapacidad de las $y$ los infantes para tomar decisiones libres y razonadas $y$ es, por tanto, no consensuada por definición ${ }^{3}$ (Frank et al., 2010, p. 875).

Si bien este nuevo enfoque sobre las libertades individuales y los derechos universales criminaliza más severamente el abuso sexual e infantil, precisamente por atentar contra la libertad y el derecho a la autodeterminación, también generó una serie de reformas que disminuyeron la edad de consentimiento, las cuales obedecían al reconocimiento de la libertad de las personas para gozar de su sexualidad (Frank et al., 2010).

A partir de la década de los sesenta, además, se observan dos fenómenos que entran en tensión: por un lado, el segundo movimiento feminista logró que se endurecieran las reformas para criminalizar la violación sexual (Cocca, 2015) y, por otro, se empezó a observar una relación entre la pobreza y el embarazo adolescente (Ehrlich, 2006). Las reformas orientadas al endurecimiento de las penas por violación introdujeron rangos de edad para definir las relaciones impropias.

3 Traducción propia. 
De esta forma, se buscaba proteger a las menores de edad de hombres abusivos, sin que se diera una negación absoluta de su sexualidad, pues los rangos de edad introducían una flexibilidad para que las adolescentes establecieran relaciones con muchachos de edades similares (Cocca, 2015; Sutherland, 2003). Esto suponía, claramente, un reconocimiento de la sexualidad como una esfera de la vida de las personas menores de edad. No obstante, este reconocimiento de la sexualidad entraba en conflicto con la preocupación por la prevalencia del embarazo adolescente en la población pobre y, más particularmente, con los esfuerzos por imponer la abstinencia entre la población adolescente como mecanismo para evitar el embarazo. La prevalencia del embarazo adolescente supone una presión sobre los sistemas de asistencia social, los cuales recurren a la popularización de programas anticonceptivos para combatir las mayores tasas de natalidad observadas en los grupos más pobres. El embarazo adolescente, especialmente aquel vinculado con la pobreza, se convierte, además, en un símbolo visible de comportamientos sociales censurables, tales como la pobreza misma, la irresponsabilidad, el abandono, el egoísmo, la ignorancia y la promiscuidad y que dan pie a un nuevo llamado, por parte de la derecha, para denunciar la pérdida de valores. Se retoma así, el argumento de que, para proteger la moral social, se debe promover la abstinencia adolescente, lo que supone la negación de la sexualidad de este grupo etario, particularmente en las mujeres. Esto último se reforzó en 1981 en Estados Unidos, cuando la Corte Suprema de Justicia justificó la existencia de diferentes edades de consentimiento para mujeres $y$ hombres con base en el riesgo de embarazo que la actividad sexual supone para las mujeres (Sutherland, 2003). Lo anterior permitió que, a partir de la década de los noventa, el control de la sexualidad adolescente $y$, en especial las campañas para promover la abstinencia sexual, cobrara fuerza como un mecanismo de control de la natalidad (Cocca, 2015). Por ejemplo, el Fondo Internacional de las Naciones Unidas para la Infancia promueve su ABC para la protección sexual como "Abstain. Be faithful. Use a Condom" (abstención, fidelidad y uso de condón), donde se promueve que "el sexo más seguro es no tener sexo del todo" (UNICEF, 2018).

\section{CUADRO 1 \\ CANTIDAD DE PAÍSES, SEGÚN EDAD DE CONSENTIMIENTO Y EDAD DE CONSENTIMIENTO PROMEDIO POR REGIÓN (en años)}

\begin{tabular}{cc}
\hline $\begin{array}{c}\text { Edad de } \\
\text { Consentimiento }\end{array}$ & Cantidad de países \\
\hline Total & 194 \\
11 & 1 \\
12 & 2 \\
13 & 5 \\
14 & 32 \\
15 & 25 \\
16 & 72 \\
17 & 4 \\
18 & 39 \\
20 & 1 \\
21 & 1 \\
Debe estar casada & 12 \\
\hline
\end{tabular}

\begin{tabular}{cc}
\hline Región & Edad promedio \\
\hline Mundo & 15,9 \\
África & 16,2 \\
Asia & 16,0 \\
Europa & 15,3 \\
Norteamérica y & 16,3 \\
Centroamérica & \\
Oceanía & 15,7 \\
Sudamérica & 15,3 \\
\hline
\end{tabular}

Fuente: Murigi (2018) 
En consecuencia, en el siglo XXI se va a observar un rango más amplio de la edad de consentimiento, reflejando así tanto la presión para disminuir dicha edad con base en el argumento de la libertad sexual como la presión para aumentarla con base en la necesidad de proteger a las personas menores de edad de los delitos sexuales y el embarazo adolescente. Se tiene así, por un lado, lo que Jackson y Scott (2015) denominan libertarianismo del 'sexo positivo', que defiende que las personas menores de edad son "intrínsicamente sexuales"4 y, por otro, una preocupación por el abuso infantil que conduce a "negar o, al menos ignorar, la posibilidad de que las y los infantes sean seres sexuales" (Jackson \& Scott, 2015, p. 44). El cuadro 1 resume la edad de consentimiento en el mundo (Murigi, 2018), la cual va a variar entre los 11 y 21 años, en Nigeria y Baréin, respectivamente. Asimismo, se observa un aumento de la edad de consentimiento promedio relativa a la observada antes de la Segunda Guerra Mundial, la cual se va a ubicar en 16 años (Cocca, 2015; Murigi, 2018; Sutherland, 2003; UNICEF, 2018). Puede observarse del cuadro 1 que el 60\% de los países han definido la edad de consentimiento de los 16 años en adelante y únicamente tres países (Nigeria, Angola y Filipinas) han establecido edades de consentimiento por debajo de los 13 años. Esto implica que Costa Rica se encuentra entre los países en el mundo con una de las menores edades de consentimiento (13 años), muy por debajo del promedio de todos los continentes en el mundo. Finalmente, el cuadro también muestra que efectivamente existe una tendencia a la estandarización de las normas internacionales, ya que, en todos los continentes, la edad de consentimiento promedio ronda los 15 o 16 años.

La tensión existente entre ofrecer anticoncepción a las personas adolescentes y validar, mediante esa política, la supuesta pérdida de moralidad se resuelve devolviéndole el control de la sexualidad adolescente a los progenitores, mediante el requerimiento de avisarles de la provisión de anticonceptivos a sus hijas (Ehrlich, 2006). Tanto esto, como el origen mismo de las leyes relativas a la edad de consentimiento, suscitan críticas a dichas leyes por parte de autores que las denuncian como un instrumento que busca negar la autonomía (Ehrlich, 2006), administrar y regular el sexo de las personas adolescentes (Janssen, 2015) o perpetuar el poder represivo y el control del cuerpo de las mujeres (Sutherland, 2003). En particular, estas y estos autores critican que las leyes introducen un margen de discrecionalidad que le permite a la sociedad aplicar la ley para imponer sus valores $y$ mantener el statu quo. Por ejemplo, Sutherland (2003) señala que las leyes pueden ser usadas por los progenitores para evitar que sus hijas o hijos salgan con personas de otros grupos sociales; se toleran más las relaciones abusivas cuando es una mujer quien se involucra con un adolescente; existe evidencia de que a las mujeres en ciertos ámbitos se les obliga a casarse con el hombre que las violó para proteger sus intereses; o se aplican las leyes en forma diferente para los diversos grupos sociales, argumentando diferencias culturales o étnicas, al punto que se ha defendido el abuso y la violación de adolescentes latinas en Estados Unidos, argumentando que estas son más promiscuas y están condicionadas culturalmente a iniciarse sexualmente en edades tempranas. La discrecionalidad en la aplicación de las leyes también se observa en el uso del argumento de la promiscuidad, según el cual un crimen no puede ocurrir si la mujer no es "honrada", o en alegar ignorancia sobre la edad, lo que exonera de castigo a quienes aducen haber desconocido que la persona con quien sostuvieron relaciones era menor de edad (Horvath \& Giner-Sorolla, 2007). Este último argumento sigue siendo una defensa legítima en algunos lugares, tales como el Reino Unido, donde es aplicable cuando se ha violado a menores entre 13 y 16 años (Horvath \& Giner-Sorolla, 2007). Desde esta perspectiva del libertarianismo 'sexo-positivo', las leyes relacionadas con la edad de consentimiento se constituyen en un instrumento para imponer la visión del poder parental y mantener el orden social, pues buscan definir los parámetros para normar el sexo entre adolescentes y, por tanto, su sexualidad, mediante la imposición de la abstinencia. Esto le niega la sexualidad a la persona adolescente hasta que alcance

4 Traducción propia. 
esa edad de consentimiento (Sutherland, 2003), lo cual no es deseable, pues obvia características asociadas al desarrollo natural de la persona (Bullough, 2005).

Tal vez uno de los ejemplos en donde más claramente se ilustra cómo la edad de consentimiento estaría operando en contra de grupos considerados desviados o anormales sea el caso del Reino Unido, donde se estableció una edad de consentimiento mayor para la homosexualidad masculina, i.e., 21 años en lugar de 16 (Ellis \& Kitzinger, 2002; Waites, 2003). Si bien la edad de consentimiento se igualó en 2000 , el caso puso en evidencia una serie de contradicciones respecto al tema: en primer lugar, existía una inconsistencia al fijar la edad de consentimiento por encima de la edad en que una persona es criminalmente responsable, pues esto sembraba dudas sobre cuándo se alcanza realmente el grado de racionalidad necesario para responsabilizar a una persona de sus decisiones (Waites, 2003). Y, en segundo lugar, el sexo entre menores con 16 a 18 años estaba descriminalizado, lo que suponía un reconocimiento de que estos adolescentes sí estaban facultados para consentir; pero si uno de ellos se relacionaba con alguien mayor, entonces esa otra relación sí era castigada (Waites, 2003). Finalmente, se logró disminuir la edad de consentimiento para el sexo homosexual masculino a los 16 años para equipararlo con el sexo heterosexual $y$ homosexual femenino con base en argumentos de igualdad $y$ derechos humanos.

En el fondo, el problema reside en la existencia de dos campos de argumentación, además no necesariamente explicitados: el de la racionalidad y la libertad de los individuos y el de la moralidad de la sociedad. En el primero, la dificultad radica en reconocer una idea de libertad para consentir sexualmente y supone la idea según la cual los individuos alcanzan en algún momento un grado de madurez equivalente a una racionalidad en la decisión. Dentro de este campo, se ha pasado de regular la relación entendida como una matriz patrimonial, donde la mujer es propiedad y el varón dueño y juez de sus dominios y decisiones, en términos generales, a la idea de regular la libertad sexual de los individuos. En el segundo, la cuestión radica en establecer los límites de las prácticas sexuales, es decir, admitir el contenido de las costumbres -"las buenas costumbres"-, aceptar prácticas individuales que socialmente no han sido admitidas o no lo son aún. En este segundo elemento, la sociedad expresa con alguna dificultad la conciencia sobre lo que es admisible para sí misma y lo que no. Así, el primer campo se traduce en un enfoque sobre las consecuencias jurídicas de los actos individuales, de suerte que ante la conducta del individuo se pueda determinar si realmente está actuando y los daños y perjuicios resultantes le puedan ser atribuidos o no a ese individuo. Este enfoque busca determinar la responsabilidad del "agente" frente a los demás y viceversa. En cambio, el segundo campo se traduce en una visión según la cual no solo se regula lo socialmente admisible, sino que se pueden proteger intereses específicos, como la integridad de la o el infante, independientemente del primer enfoque.

Entre ambos campos, entre libertad sexual e integridad física, queda el problema del lugar de la construcción del consentimiento, sobre la cual regresaremos posteriormente. Se añade a todo esto que la diferencia etaria produce una imagen socialmente negativa. Ambos campos juegan sobre la representación de racionalidad y sobre cómo se expresa en la protección de los intereses y libertades individuales. La defensa de la integridad de las personas infantes recubre la defensa de lo que la sociedad se representa como admisible. De hecho, la estandarización y universalización de las normas que se observa a lo largo del siglo XX responde a esta posición desde los derechos humanos, la cual permite el surgimiento de las instituciones internacionales referidas anteriormente por Frank, Camp y Boutcher (2010). Como ya se mencionó, esta perspectiva de derechos humanos difiere substancialmente de la visión libertaria en tanto busca la protección de la persona menor de edad. Así, la Convención sobre los derechos de la niña y el niño de Naciones Unidas, firmada en 1989, establece en su artículo 19 la protección de "toda forma de violencia física o mental, daño o abuso, negligencia o trato negligente, maltrato o explotación, incluyendo el abuso sexual” (OHCHR, 1989), mientras que los artículos 34 y 35 de la misma convención otorgan la responsabilidad a los Estados miembros de proteger a la persona menor de edad de "de toda forma de explotación sexual y abuso 
sexual" y del rapto, venta o tráfico, respectivamente. Estos derechos, además, se fundamentan en el reconocimiento de un cuidado y asistencia especial a la infancia, justificada en su inmadurez física y mental, que emana de la Declaración Universal de los Derechos Humanos de 1948 y de la Declaración de los Derechos de la Niña y el Niño de 1959 (OHCHR, 1989).

Por su parte, la información estadística apoya la postura de derechos humanos, mas no así la posición libertaria del 'sexo-positivo'. Por ejemplo, esta última argumenta en contra de las leyes relativas a la edad de consentimiento, aduciendo que estas criminalizan relaciones entre personas de edades similares una vez que una de ellas alcanza la edad de consentimiento. A estos, se les conoce como casos del estilo Romeo y Julieta. Sin embargo, un estudio estadístico realizado por Bierie y Budd (2018) con información policial recabada durante veinte años en más de 60000 instancias policiales en Estados Unidos muestra que la violación estatutaria está lejos de ser la norma, pues representa apenas el 5\% del total de crímenes sexuales. Y los casos Romeo y Julieta son aún menores, representando apenas el 0,8\% del total de crímenes sexuales (Bierie \& Budd, 2018). Sin embargo, los casos de violación estatutaria presentan características típicas de violencia que deben regularse y castigarse: en primer lugar, la probabilidad de observar formas adicionales de agresión sexual se incrementa conforme aumenta la diferencia de edad entre la víctima y el ofensor. En segundo lugar, conforme la diferencia de edad aumenta es más probable que el acto sea involuntario o forzado, de forma que conforme esta crece, el tipo de relación reportada pasó de ser romántica o de pareja a ser una relación con una persona conocida, extraña o con una persona que ostenta una relación de poder respecto a la víctima (Bierie \& Budd, 2018). En tercer lugar, conforme aumenta la diferencia de edad entre la víctima y su agresor, se incrementa la probabilidad de que la menor de edad inicie su actividad sexual a una edad más temprana (Bierie \& Budd, 2018), lo que supondría un comportamiento más depredador por parte del agresor. En cuarto lugar, conforme aumenta la diferencia de edad, se incrementa la probabilidad de un embarazo también a una menor edad (Cocca, 2015; Bierie \& Budd, 2018). Y, quinto, los autores identifican que la mayoría de las víctimas son mujeres con hombres agresores, lo que evidencia un problema de género que no puede obviarse en nombre de la libertad sexual. En promedio, encuentran que esta diferencia de edad es de 9,8 años, con una edad promedio de la víctima de 13,9 años (Bierie \& Budd, 2018).

A pesar de lo anterior, la violación estatutaria está permeada por la valoración social de este tipo de relaciones, la cual tiende a ser ambivalente $y$, finalmente, permisiva. Al respecto, Horvath y Giner-Sorolla (2007) realizaron un experimento para medir la valoración de las relaciones entre personas adultas y menores de edad y encontraron que, en promedio, se valora más negativamente dicha relación (i.e., se considera peor y más dañina) cuando la persona adulta es un hombre o conforme se incrementa la diferencia de edad entre las personas involucradas. Las relaciones homosexuales con una persona menor de edad, por su parte, no fueron valoradas en el experimento en forma diferente a las relaciones heterosexuales. Además, los autores encontraron que las mujeres son más severas a la hora de juzgar este tipo de relaciones que los hombres, lo que sugiere la existencia de diferencias asociadas al género. No obstante, si bien casi el $90 \%$ de las personas participantes en el experimento reconocieron cuándo se comete un delito sexual por mantener relaciones con menores de edad, apenas algo más de la mitad de estas personas estaba dispuesta a imponerle un castigo a la persona por ese delito. E incluso cuando imponían dicho castigo, se inclinaban mayormente por una pena con libertad condicional, no con la ejecución de la pena en la cárcel. Esto evidencia que, si bien se reconoce el delito, existe una alta permisividad por parte de la sociedad que admite la persistencia de este tipo de relaciones con menores de edad.

Al respecto, Jackson (1978) sostiene que la violación no puede entenderse separada de los modos convencionales de las expresiones sexuales, pues la identidad de género y la construcción de esos scripts sobre el significado de la feminidad o la masculinidad facilitan las situaciones en las que se materializa la violación como una ocurrencia típica de las relaciones en la sociedad. Así, la necesidad de validar la masculinidad y de entender los deseos masculinos como un poder o un 
impulso que domina al hombre lo exculpan de cualquier responsabilidad frente al acto de violación. Al mismo tiempo, la identidad de género permite incorporar motivos no sexuales en la justificación de la violación: por un lado, se valida la masculinidad y la necesidad de satisfacer el deseo masculino y, por otro, se acepta la violación como un mecanismo para exigir luego el "amor", apoyo y protección de un hombre. Así, se objetiva la sexualidad de la mujer para intercambiarla a cambio de protección material (Jackson, 1978). Por ejemplo, una familia podría acceder a entregar a su hija menor de edad a un hombre mayor a cambio de que este se constituya en proveedor y garantice que va a mantenerla y "asegurarle" así la vida.

Esta rápida mirada histórica nos muestra por una parte un objeto variable de regulación y, por otra, una modificación en la manera de concebir la regulación y las prácticas sexuales. Se ha pasado de un objeto considerado como propiedad a la consideración de la libertad sexual de las personas. Y en el camino, se ha pasado de considerar las formas de agresión sexual como la destrucción de un bien (bien de intercambio, de honor, etc.) a una violación de la libertad sexual de cada cual, considerada como un "bien jurídico" (susceptible, pues, de ser dañado o tutelado). Y existe un problema de percepción de lo que significa la práctica sexual con una persona menor, tanto por parte de la persona agresora como por parte de la víctima.

El hecho de que regularmente la edad para los esponsales de la mujer sea inferior a la de los hombres conduce a pensar que, si la edad es un criterio de madurez intelectual para decidir, supondría una más temprana madurez en la mujer que en el hombre y, sin embargo, no se traslada este criterio a otras disposiciones, por lo cual el campo de acción de la mujer se ve, por el contrario, reducido. Hoy en día, la misma edad ha, en principio, resuelto el problema de la desigualdad sobre la minoridad, pero no el enfoque de la libertad individual y el del interés por proteger la infancia. Quedaría por supuesto discutir si la madurez intelectual se puede reducir a la madurez reproductiva.

Ambos enfoques, tanto el de la racionalidad y libertad de los individuos como el de la moralidad de la sociedad, tienen un cierto arraigo en los derechos humanos, pues el primero supone precisamente que el consentimiento y el carácter sexuado de los individuos se inscriben en la libertad individual. Y el segundo contempla la libertad y la seguridad de los individuos considerados aún incapaces para protegerse por sí solos. Es evidente que el primer enfoque, al no ir al extremo de liberar todos los cuerpos y al aceptar un límite para el consentimiento -por muy bajo que seaacepta que existe un rango etario bajo el cual no es posible legalmente el consentimiento, porque se supondría que no hay "entendimiento libre" sin poder determinar una regla específica. Juega con un fantasma social sobre lo que significa la madurez sexual $y$ la madurez intelectual.

Es obvio que o bien la regla debería considerar, caso por caso, la madurez de los individuos, dejando a posteriori, el establecimiento de la licitud o ilicitud de las relaciones sexuales, o bien debería enunciar una regla general para todos los casos que fije las relaciones sexuales socialmente admisibles. Esta segunda opción es la históricamente adoptada de manera general, porque evita las dificultades del "caso por caso" y del a posteriori de la primera opción, pero según los dos enfoques anteriores, se mostraría arbitrario en la medida en que científicamente no pueda establecerse la edad de la madurez intelectual de cada cual. Aquí la madurez no haría simplemente referencia a un coeficiente intelectual, sino a la capacidad de discernir las condiciones de sujeción en las que el individuo decide. El primer enfoque apuntaría, en cambio, a una licencia con un examen para obtenerla, a la manera de la licencia de conducción y, al igual que esta, supondría un mínimo de edad para poder solicitarla. Evidentemente, esta vía es impracticable en la medida en que el control de conducción en carretera supone un fácil acceso al espacio público y la posibilidad de constatar la posesión de dicho permiso, mientras las relaciones sexuales no suelen tener lugar mayoritariamente en los espacios públicos, sino en el ámbito privado, de mucho más difícil acceso o control. La idea de la obtención de la licencia apuntaría al carácter individual de la madurez. En cualquier caso, habría en ambos enfoques un mismo fantasma teórico, un supuesto no enunciado: que la madurez reproductiva se confunde con la madurez intelectual, como se verá a continuación. 


\section{EDAD DE CONSENTIMIENTO EN COSTA RICA}

En esta sección, hacemos una revisión histórica de la normativa legal que ha regido la edad de consentimiento en Costa Rica. Para ello, fueron consultados los diferentes códigos penales que ha tenido Costa Rica. La edad de consentimiento ha variado de manera similar a las variaciones observadas en el mundo. Asimismo, la edad de consentimiento actual en Costa Rica ha disminuido con respecto a los niveles del siglo XIX, por ejemplo, a la vez que la ley permite múltiples rangos de edad que introducen un considerable margen de permisividad en el establecimiento de las relaciones sexuales con personas menores de edad. No se trata de reconstruir la historia de la normativa penal. Nos interesa poder poner en contexto el uso de la edad del consentimiento, en particular sobre la percepción que se tiene comúnmente pero también legalmente.

Las normas penales que rigen en el Estado costarricense durante la mayor parte del período previo a la constitución de la República fueron heredadas de España y están plasmadas en el texto intitulado Las siete partidas de don Alfonso X el Sabio, haciendo alusión a su autor, el rey Alfonso $\mathrm{X}$ de Castilla, quien gobernó esa región durante 32 años en el siglo XIII. Como es de esperarse, el texto es conforme a los lineamientos de la Iglesia Católica y fue publicado entre 1256 y 1265. En la Partida Cuarta, Título 2: De los casamientos, la ley 5 establece el consentimiento verbal como criterio para contraer matrimonio y la ley 6 define dicha facultad como tener "entendimiento sano para consentir el casamiento y que son tales que no tienen embargo que les impida yacer con las mujeres [...]" (Alfonso X el sabio, 2006). De lo anterior, la facultad de entendimiento parece confundirse con la capacidad para engendrar, de manera que se prohíbe el casamiento para quienes son estériles.

Además, debe notarse que el texto no introduce una edad de consentimiento claro y explícito, como sí lo hizo el Estatuto de Westminster de 1275, por poner un contraejemplo, sino que el criterio que establece es natural y está explícitamente asociado a la capacidad reproductiva de cada individuo. Por su parte, el Título 14: De las otras mujeres que tienen los hombres que no son de bendiciones, de la misma partida permite tener "barragana" (i.e., amante) si esta no es virgen y no es menor de 12 años, mientras que la ley 1 del título 16 de la Partida Sexta, que regula la administración de los bienes de las personas huérfanas, establece la tutela para personas menores de 12 años en el caso de las mujeres y 14, en los hombres, lo que sugiere que esta es la minoría de edad de la época, aunque esta no está explícitamente definida. Lo anterior implica que se consideraba que la mujer alcanzaba la madurez antes que los hombres, partiendo de la idea de que la edad reproductiva de la mujer es más temprana, pero al igual que en el caso de los otros países haría referencia explícita a la madurez reproductiva y no necesariamente a la madurez intelectual de los individuos. Es evidente que, por una parte, se regulan los cuerpos en su capacidad biológica en vistas a la reproducción $y$, por otra, que la capacidad legal entendida como madurez intelectual no aparece claramente.

En 1841, bajo la administración de Braulio Carrillo, se promulga el Código General del Estado de Costarrica. En él, se establece como requisito para los esponsales una edad de 10 años, el consentimiento de los progenitores y escritura pública (Código General del Estado de Costarrica, 1841, título V, art. 85). No obstante, el matrimonio no puede consumarse hasta que la mujer alcance los 12 años y el hombre, 14 (art. 88), ambas partes expresen un mutuo y libre consentimiento (art. 89) y se cumpla lo prescrito por el Concilio de Trento (Código General del Estado de Costarrica, 1841, título V, art. 100). Además, el artículo 102 permite a las o los tutores la oposición al matrimonio si existe una "desigualdad notable entre los dos pretendientes en la edad que exeda [sic] de treinta años por lo menos". Por tanto, este código introduce por primera vez la edad de consentimiento en Costa Rica en conformidad con la normativa vigente internacionalmente (i.e., el Concilio de Trento), le da potestad a las o los tutores de aprobar el matrimonio y establece 
un rango, bajo el cual la edad de consentimiento puede anularse. Asimismo, debe señalarse que esta edad de consentimiento es significativamente inferior a la edad de mayoría de edad, la cual se estableció en 25 años (título X art. 192). El código también tipifica los delitos contra el honor: específicamente, la violación para mujeres menores de 12 años y el estupro para mujeres mayores de 12 y menores de 17 (art. 419). El artículo 419 establece un rango de penas diferenciadas por el "uso deshonesto" según la edad de la víctima: menos de 12, a una pena entre uno y cuatro años de cárcel; entre 12 y 17 entre uno o dos años (aquí además debe intervenir la violencia en la comisión del delito). El 420 hace referencia a las mujeres honestas de más de 17 años, cuyo abuso se castiga con la misma pena del 419. Ahora bien, este artículo 420 interesa porque señala un rango de edad (mayor de la pubertad y menor de 17 años) de la mujer con la cual hubiese "cópula carnal"5. Este es el supuesto de un cierto consentimiento.

Las diferencias fundamentales de ciertas figuras penales radican en que se sanciona objetivamente una conducta o sin consentimiento o con consentimiento. Al ser la pena menor en el caso de haber consentimiento, se reconoce una edad después de la cual la persona es considerada en un grado capaz de discernir (primer enfoque), pero a la vez, aun consintiendo la integridad de la persona sigue protegida, acaso contra su voluntad (segundo enfoque).

Durante la administración de Tomás Guardia, se promulga el Código Penal de la República de Costa Rica (1880). En él, se mantiene la edad de consentimiento en 12 años. Por su parte, se tipifica como violación el acto sexual con mujeres menores de 12 años (art. 382). El estupro inicialmente se castigaba cuando la involucrada era una "doncella mayor de doce años y menor de veinte cuando medie engaño" (art. 384). Posteriormente, se elimina la condición de haber sido engañada en la reforma al artículo de 1917, lo cual refleja un endurecimiento de la posición en contra del estupro. Este código, además, criminaliza el rapto de mujeres, castigando más severamente al infractor cuando la doncella es menor a 12 años o de buena fama que cuando esta tiene edades entre los 12 y 20 años (art. 379). En todos los casos, se suspende el proceso penal si se da el consentimiento para casarse, lo cual se entiende debido a que el delito está estipulado como un delito contra el honor, de forma tal que, al haber una promesa de matrimonio, se reestablece dicho honor para la familia ofendida. Debe subrayarse, además, que el rango de edades que se protegen con esta legislación es amplio y abarca de los 12 a 20 años.

La cuestión de la seducción (art.420, CGEC 1841) o del engaño (art.384, CP 1880) interesa en la constitución de la idea de consentimiento, porque hace referencia a la competencia misma para discernir los fines de la relación ("objetivo con la persona") y consecuentemente habla de su facultad. Asimismo, es un punto a tratar en los vicios del consentimiento, en la medida en que supone la nulidad de un acto jurídico, particularmente en materia contractual. Este tipo de artículo pone en evidencia un cierto carácter contractual, gracias a una patología en la voluntad de una de las partes (engaño), pues el engaño supone aceptar una acción -¿acaso una prestación?- en vistas a algo -¿̇otra prestación?-. Existiría una intuición natural sobre el carácter contractual de las relaciones sexuales $y$, por extensión, del emparejamiento.

Como se ha anticipado ya, hacer descansar estas figuras jurídicas en el consentimiento supone que no hay un contexto socioeconómico que influya clara o subrepticiamente en los actores en el momento de consentir. Así, parece que el ceder se traspone al consentimiento (Fraisse, 2017, 61ss). El consentimiento necesita condiciones explícitas sobre lo que se consiente (para nada sobreentendidos, cosas no dichas, cosas calladas o tácitas), supone pues que dichas condiciones sean claras sobre lo que es común en la iniciación sexual, sobre lo inaceptable, sobre las expectativas que emergen en la relación, etc.

5. En todos los casos, hay equivalencia de cárcel con el pago de una multa, sin considerar los daños producidos. 
Hacia inicios del siglo XX, y paralelo a los cambios observados en el resto del mundo, se da un incremento de la edad de consentimiento de 12 a 15 años, con la aprobación del Código Penal de Costa Rica de 1918 de la administración de Federico Tinoco. En esta nueva legislación, la violación se establece al abusar de personas menores de 15 años (art. 278), de manera que se introduce la protección a "personas de uno u otro sexo" y no ya solo la de las mujeres, lo que significa un avance en materia de protección de la niñez. El estupro, por su parte, castiga "el acceso carnal con persona mayor de quince años y menor de diez y ocho" (art. 279), mientras que el rapto continúa siendo definido únicamente cuando la ofendida es una mujer. En este caso, el rapto se castiga igual que la violación cuando la ofendida es menor de 15 años y con prisión (pero menor sentencia) cuando esta tiene entre 15 y 21 años, aun cuando la mujer haya estado anuente a que se realizase el rapto (art. 286). Lo anterior supone un mayor interés por proteger a la mujer, pues no se considera la voluntad de esta para castigar el acto. No obstante, y manteniendo las políticas anteriores, todos los procedimientos se suspenden en caso de que haya consentimiento para casarse.

El Código Penal de 1924, publicado durante la administración de Julio Acosta, mantuvo la edad de consentimiento en 15 años (art. 300) e impone castigos más severos cuando la violación es cometida por familiares o personas con poder sobre la víctima (art. 305). En 1928, esto último es reformado y se establece la mayor pena para cuando "resultare grave daño en la salud de la víctima o se cometiere el hecho por un sacerdote o ministro de cualquier culto o por un encargado de la educación o de la guarda del ofendido u ofendida, o con el concurso de dos o más personas". Por su parte, el estupro no solo se mantiene para resguardar a las mujeres entre 15 y menos de 18 años, sino que se amplía para proteger a aquellas con edades entre 18 y menos de 21 "siempre que haya mediado promesa matrimonial o cualquier modo de seducción por engaño” (art. 302). Y, el rapto se sigue castigando hasta los 21 años, con mayores penas cuando la ofendida es menor de 15 (art. 309). En forma similar a los códigos anteriores, se suspende el procedimiento contra todos estos delitos contra el honor cuando se da el consentimiento para casarse.

El honor de las mujeres es particularmente un bien protegido y regulado, en vistas a su emparejamiento. Casi todos los artículos hacen referencia a la figura de "mujer honesta" y, en contra partida, "mujer pública": marcando claramente que el honor y honestidad de la mujer se protege como garantía del objeto que se transfiere, y en cambio la mala fama tiene como consecuencia la reducción de las penas en la violación o en el estupro, pues el bien aparece considerado implícitamente como dañado, luego la protección es más reducida.

El Código Penal de 1941 disminuye la edad de consentimiento a los 12 años e introduce la figura del Patronato Nacional de la Infancia como ente que debe velar por la protección de las personas menores de edad víctimas de abuso. Además, se libra de toda responsabilidad a las personas menores de edad cuando estas hayan sido seducidas por una mayor de edad (art. 226). La violación por contar con una edad menor a la de consentimiento ocurre cuando la víctima es menor de 12 años (art. 216) y hay un agravante cuando el crimen lo comete un familiar o una persona con poder sobre la víctima (art. 218). En 1959, se reformó, además, este artículo para impedir el indulto a quien haya violado a una menor de 12 años y se endurecieron las penas relacionadas con la violación. El rango de edad para el estupro mantiene la diferencia para un rango de 6 años, pero reduce las edades, de forma que se penaliza cuando la ofendida tiene entre 12 y 15 años y entre 15 y menos de 18, cuando haya habido engaño de por medio (art. 219). Lo mismo ocurre para el rapto, el cual se castiga cuando la ofendida es menor de 18 años y en forma más severa cuando se es menor de 12 . Nuevamente, todo procedimiento se suspende si la ofendida accede al matrimonio con su ofensor (art. 225).

El actual Código Penal mantiene la edad de consentimiento en 12 años (art. 156) e introdujo penas para quien autorice o case a personas menores de 15 años (art. 180-181). Este código reduce aún más el rango de edad de la ofendida para el estupro, el cual se define únicamente para edades entre 12 y 15 años (ya no entre 12 y 18 años) cuando la ofendida es "mujer honesta", 
independientemente de que esta haya consentido al acto (art. 159), de forma que se reintroduce el énfasis sobre la honorabilidad de la mujer. Finalmente, el rapto se reclasifica como "rapto impropio" $y$ castiga a quien "raptare con fines libidinosos a una mujer honesta mayor de doce y menor de quince años, con su consentimiento" (art. 164), de forma que también se observa una reducción de las edades de la ofendida, a la vez que se perdona el rapto si la ofendida se casa con su raptor (art. 165). Y, ¿qué sentido tiene hablar de "rapto con consentimiento"? Implícitamente sobrevive la regla del consentimiento parental, el cual no se substituye por el consentimiento de la víctima. El matrimonio substituye la aparente oposición entre la víctima y el victimario, al pretender borrar la ofensa. Tiene una función opresiva bastante particular, porque el consentimiento al matrimonio (ius connubii) substituiría y corregiría la ausencia de consentimiento del acto precedente (vicio del consentimiento). Normalmente, se deja de lado que esa corrección busca "solventar" el problema de honor de la familia de la víctima y empuja a la víctima a una posición socialmente en la que no puede considerarse más que oprimida por las circunstancias. La potestad de otorgar perdones también se les otorga a las personas ofendidas y al Patronato Nacional de la Infancia (art. 81). Para todos los crímenes, se mantiene un castigo más severo en caso de que el ofensor sea pariente u ostente algún poder sobre la víctima.

Posteriormente, la ley 7899 Ley contra la explotación sexual de personas menores de edad viene a modificar los tipos penales relacionados con los delitos sexuales. Entre ellos, establece cárcel entre 10 y 16 años a quienes mantengan relaciones sexuales con personas menores de 12 años, la persona sea incapaz de resistir o se emplee violencia corporal o física. Las penas para este tipo de delitos se reducen a entre 2 y 6 años si las víctimas tienen edades entre 12 y menos de 15 años. Además, se castigan otros delitos tales como aquellos relacionados con la producción y distribución de pornografía infantil, proxenetismo, trata de personas o corrupción de menores.

La ley 9406 Fortalecimiento de la protección legal de las niñas y las adolescentes mujeres ante situaciones de violencia de género asociadas a relaciones abusivas, que entró a regir en 2017, eleva la edad de consentimiento de 12 a 13 años y modifica los rangos de edad para la pareja de las personas menores de edad, pero mayores de 13 años, en que es permitido sostener relaciones sexuales. Así, se considera violación la relación sostenida con una persona menor de 13 años. No se considera que exista consentimiento cuando se tiene entre 13 y menos de 15 años y la otra persona le lleva 5 años o más a la menor de edad (C.Penal. art, 159) o cuando se tiene entre 15 y menos de 18 años y la otra persona le lleva 7 años o más a la menor de edad.

Esta ley introduce varios elementos de análisis: primero, mantiene la diversidad de rangos e introduce las diferencias de edad; segundo, el consentimiento está asociado explícitamente a las edades de sus parejas o abusadores, lo cual podía ser el caso anteriormente, pero no se enunciaba. Regresamos al supuesto de un engaño por parte del sujeto mayor, bajo la lógica que cuán mayor sea, más podría engañar o agredir al sujeto menor. La diferencia de edad parece significar el incremento en la diferencia en la capacidad de discernir de los sujetos, y reduce subjetivamente el significado del consentimiento a una relación de fuerzas entre ambos.

Surgen varios problemas: primero, la ley se afinca en el consentimiento, con lo que supone que el consentimiento es suficiente para dirimir cualquier problema. Ignora, por ejemplo, que el consentimiento suele confundirse por una parte con una "expresión clara de la voluntad" y, por otra, con no oponer resistencia a una situación. Pero quien está en una situación de sujeción u opresión difícilmente expresa claramente su voluntad ni se manifiesta contrariamente, pues ni encuentra el modo, ni las fuerzas para ello, si no es el caso que la opresión aparezca como naturalizada, con lo cual es imposible apreciar cualquier vicio en el consentimiento. Además, ¿no debería el consentimiento estar establecido objetivamente y ser definido en función de la capacidad del sujeto y no de la diferencia de edades? 


\section{DATOS Y MÉTODOS}

En las secciones anteriores, presentamos de manera somera la historia y los antecedentes respecto de los delitos sexuales, sobre cuya noción discutimos los alcances y las implicaciones jurídico-filosóficas. Ahora, analizaremos los datos de la Encuesta Nacional de Salud Sexual y Reproductiva. Este ejercicio supone cuantificar los delitos sexuales para poder visualizar su significado. Para ello, se utilizó la Encuesta Nacional de Salud Sexual y Reproductiva (CCP, 2010 y 2015) para los años 2010 y 2015. Estas encuestas recopilan información de mujeres y hombres con edades de 15 años y más sobre su comportamiento sexual e historial reproductivo, incluida información sobre la edad de la pareja en su primera experiencia sexual y los últimos doce meses anteriores a la encuesta. Esto permite identificar a aquellas personas que estuvieron en una relación impropia según los términos definidos por la ley 9406 en estas etapas de su vida. Lastimosamente, la encuesta no reconstruye todo el historial sexual de las personas entrevistadas, por lo que cualquier indicador que se construya al respecto indudablemente será una subestimación del nivel real de los delitos sexuales. A pesar de ello, el dato es relevante porque, aunque imperfecta, permite una primera cuantificación de este indicador.

La encuesta de 2010 entrevistó a 1596 mujeres y 1601 hombres, para un total de 3197 observaciones, mientras que la de 2015 cuenta con 3216 observaciones: 1677 mujeres y 1539 hombres. Debe señalarse que la encuesta de 2015 no incluye una variable para los pesos poblacionales. Se hizo la consulta respectiva, pero no fue posible identificar los pesos, por lo que los resultados aquí presentados no contienen ponderaciones $y$ deben ser interpretados como representativos de la muestra. Dada la metodología de la encuesta, no existe motivo para pensar que haya un sesgo de selección o que los datos de la muestra difieran considerablemente de los valores poblacionales. Las observaciones con valores faltantes, respuestas incoherentes o valores extremos fueron excluidas, de forma que la muestra balanceada cuenta con 5668 observaciones (88\% de la muestra inicial). De estas, 51,52\% corresponden al 2010 (2920 registros) y 48,48\% al 2015 (2748 registros). No hay evidencia de sesgos por pérdida de observaciones en la muestra balanceada: la prueba-z para la igualdad de proporciones para variables categóricas y la prueba-t para la igualdad de medias para variables continuas sugieren que todos los valores obtenidos para la muestra balanceada no son significativamente diferentes de aquellos en la muestra completa y ninguna de las variables de interés tiene más de $5 \%$ de valores faltantes, excepto por la variable sobre la diferencia de edad con la pareja en la primera relación sexual, la cual tiene un $7 \%$ de valores faltantes. No se observaron patrones que pudiesen sugerir otros posibles sesgos.

\section{Delito por relación impropia en la primera relación sexual}

Utilizando Stata 12, se construyó una variable dicotómica que toma el valor de 1 cuando se tienen relaciones sexuales con una persona menor, utilizando el criterio de edad establecido por la normativa o "relación impropia" como se le conoce popularmente ${ }^{6}$. Para ello, se identificó aquellos casos en los que la persona reportó ser menor de 13 años al momento de su primera experiencia sexual; aquellos casos en los que la primera experiencia sexual ocurrió entre los 13 y menos de 15 años, pero la otra persona era al menos 5 años mayor que quien responde; aquellos en los que la primera experiencia sexual ocurrió entre los 15 y menos de 18 años, pero la otra persona era al menos 7 años mayor que quien responde y aquellos en los que la persona era menor de edad $y$ la otra persona era un pariente, maestro o jefe $y$, por tanto, se encontraba en relación de poder con respecto a la persona menor de edad. Para la estimación, se consideró la primera experiencia sexual sin importar si esta fue oral, vaginal o anal. Los resultados de esta estimación se muestran en el gráfico 1.

6 Tal y como se mencionó en la sección anterior, la ley 9406 modifica las sanciones para quienes mantienen relaciones sexuales con menores de edad. A este delito se le conoce popularmente como relaciones impropias y es el término utilizado en este artículo. 


\section{GRÁFICO 1 \\ PORCENTAJE DE PERSONAS EN LA MUESTRA BALANCEADA CUYA PRIMERA RELACIÓN SEXUAL FUE IMPROPIA*, SEGÚN GÉNERO Y AÑO DE LA ENCUESTA}

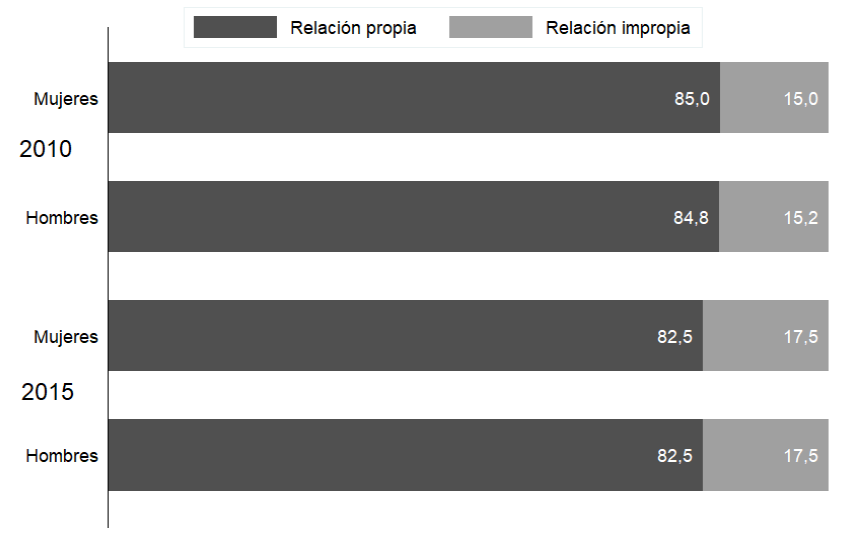

Fuente: Estimaciones propias, con base en CCP (2010 y 2015).

* Las relaciones impropias se definen conforme al criterio de edad establecido en la ley 9406 .

En total, 16,2\% de las personas en la muestra fueron víctimas de relaciones impropias según este criterio de diferencias de edad. En la muestra de la encuesta de 2010, 15,1\% de las personas fueron víctimas de una relación impropia en su primera experiencia sexual y la proporción aumentó a $17,5 \%$ en 2015, siendo esta diferencia de 2,4 puntos porcentuales (p.p.) estadísticamente significativa. Además, la proporción de quienes fueron víctimas de una relación impropia es igual para mujeres y hombres en ambos años, i.e., la prueba de proporciones para variables categóricas no muestra que la diferencia sea estadísticamente significativa. Esto es importante porque la ley 9406 se creó para la protección de las "las niñas y las adolescentes mujeres ante situaciones de violencia de género asociadas a relaciones abusivas", tal y como lo afirma su título, y aunque la ley también aplica para los hombres, los datos muestran que estos son igualmente vulnerables al abuso en relaciones impropias, por lo que el énfasis de lenguaje sobre la protección de las niñas y adolescentes mujeres podría invisibilizar la necesidad de protección de los niños y adolescentes hombres.

\section{GRÁFICO 2}

PORCENTAJE DE PERSONAS EN LA MUESTRA BALANCEADA CUYA PRIMERA RELACIÓN SEXUAL FUE IMPROPIA*, SEGÚN DIFERENCIA DE EDAD RESPECTO A LA PAREJA

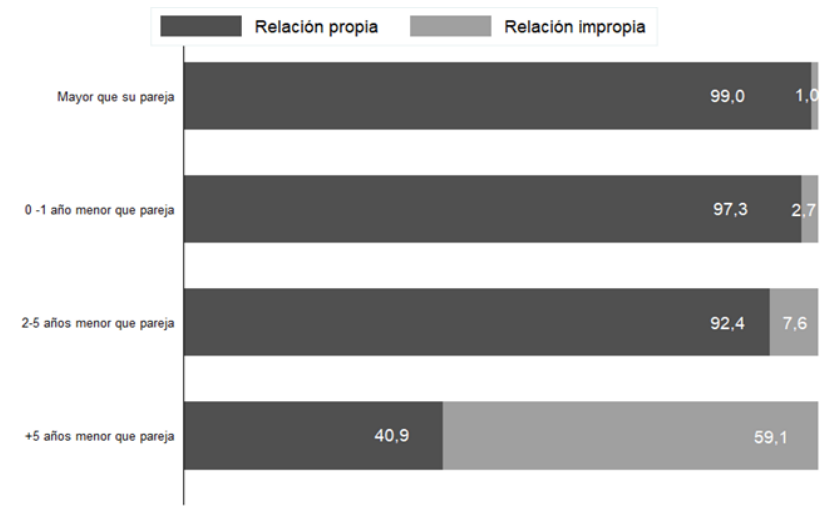

Fuente: Estimaciones propias, con base en CCP (2010 y 2015).

* Las relaciones impropias se definen conforme al criterio de edad establecido en la ley 9406. 
Por construcción, la mayor explicación de este delito es la diferencia de edad. El gráfico 2 muestra el porcentaje de la población cuya primera experiencia sexual fue una relación impropia, según la categoría de diferencia de edad con su primera pareja sexual. Como es de esperarse, casi la totalidad de casos en donde la persona reporta haber sido la de mayor edad corresponden con relaciones propias: únicamente el $1 \%$ de quienes fueron mayores que su primera pareja estableció relaciones impropias. Esto corresponde a dos casos en donde las personas tuvieron su primera experiencia sexual antes de los 13 años, por lo que las personas involucradas tenían ambas una edad inferior a la de consentimiento, $y$ otros dos casos de incesto. Similarmente, únicamente 2,7\% de quienes iniciaron su vida sexual con personas de su misma edad establecieron relaciones impropias, ya sea por ser infantes precoces o por haber sufrido relaciones incestuosas. La proporción de casos de relaciones impropias se incrementa a 7,6\% para aquellas personas que eran de 2 a menos de 5 años menores que su primera pareja sexual, lo que representa un incremento significativo respecto a los dos grupos anteriores. Sin embargo, lo más relevante es que la mayor concentración de relaciones impropias se va a observar en el último grupo: aquel donde la persona era al menos cinco años menor que su primera pareja sexual. En este grupo, 3 de cada 5 casos corresponden a relaciones impropias y los casos en este grupo representan el $81,2 \%$ del total de relaciones impropias documentadas en la muestra. Esto es consistente con los resultados encontrados por Bierie y Budd (2018), quienes sostienen que los casos de Romeo y Julieta son raros y, en su lugar, predominan aquellos con amplias brechas de edad entre las partes involucradas, lo que sirve para acentuar las relaciones de poder y las agresiones hacia menores de edad.

El cuadro 2 presenta la edad promedio de iniciación sexual y los años de diferencia promedio entre la persona que responde el cuestionario y su primera pareja sexual para los casos en que la persona ha tenido alguna actividad sexual. En primer lugar, se observa una diferencia significativa en la distribución de los diferentes grupos entre quienes sufrieron relaciones impropias y quienes establecieron relaciones propias. Como ya se mencionó, en el primer grupo, la mayor concentración de observaciones $(81,2 \%)$ corresponde a personas que eran al menos 5 años menores que su pareja, mientras que, en el segundo grupo, la mayoría de personas $(77,8 \%)$ experimentó por primera vez con alguien de su misma edad (41,3\%) o con alguien entre 2 y menos de 5 años mayor (36,5\%). En segundo lugar, la edad a la que se tuvo la primera experiencia sexual es significativamente inferior para quienes experimentaron relaciones impropias en todos los casos: esto indica un mayor grado de vulnerabilidad e inmadurez al momento de la iniciación sexual, lo cual nuevamente es consistente con Bierie y Budd (2018). Finalmente, en tercer lugar, la diferencia de edad promedio observada para quienes establecieron relaciones impropias es 4,9 veces la observada para el grupo de relaciones propias. Es decir, mientras lo típico para el grupo de relaciones propias es que la persona se haya iniciado sexualmente a los 18 años con una pareja de 20; lo típico en el grupo de relaciones abusivas es que la persona se haya iniciado a los 14 años con una persona de 24. Acaso, postergar la iniciación sexual reduce la diferencia de edad entre las personas y los expone menos a condiciones de vulnerabilidad. 


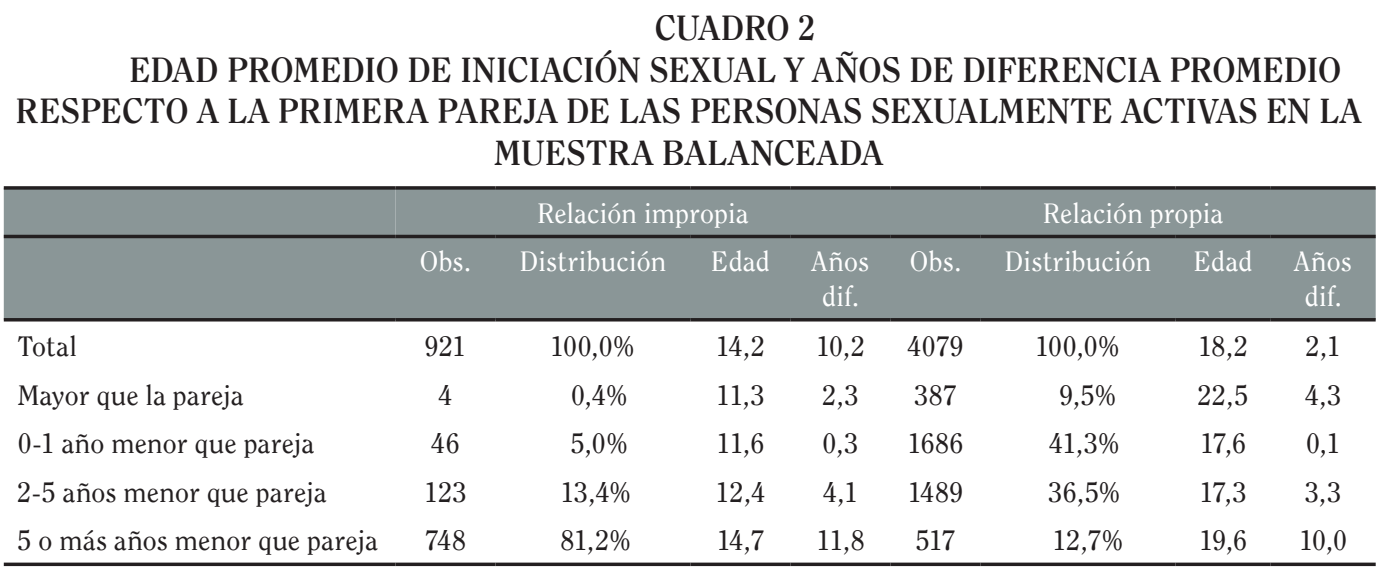

* Se excluye 668 observaciones de personas sin experiencia sexual.

Fuente: Estimaciones propias, con base en CCP (2010 y 2015).

\section{Delito por relación impropia}

Al indicador anterior se le agregó aquellos casos en donde se reportó haber estado en una relación impropia en los últimos 12 meses, con el fin de cuantificar el porcentaje que es víctima de este delito. Esto corresponde a 79 nuevos casos. Nuevamente, debe recordarse que los resultados obtenidos corresponden a una subestimación del verdadero nivel de los delitos sexuales existentes, dado que no se cuenta con el historial sexual completo de las personas, sino solo con la información para los últimos doce meses antes de la entrevista. El gráfico 3 muestra estos resultados. La muestra para la encuesta de 2010 señala que, para entonces, $15,2 \%$ de las personas entrevistadas fueron víctimas de relaciones impropias. Para 2015, este indicador había ascendido a 20,2\%, i.e., un incremento significativo de 5 p.p. Por su parte, la proporción de quienes fueron víctimas de relaciones impropias no es estadísticamente diferente para mujeres y hombres en ninguno de los dos años. Nuevamente, esto llama la atención sobre la necesidad de ofrecer apoyo y generar políticas que sean efectivas para proteger a todas las personas menores de edad de los delitos sexuales, independientemente de su género.

\section{GRÁFICO 3 \\ PORCENTAJE DE PERSONAS EN LA MUESTRA BALANCEADA QUE HAN SIDO VÍCTIMAS DE RELACIONES IMPROPIAS*, SEGÚN GÉNERO Y AÑO DE LA ENCUESTA}

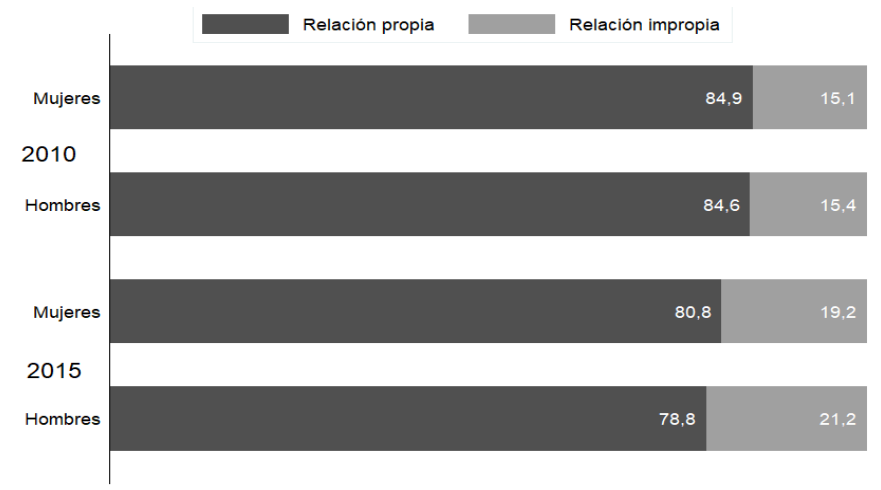

Fuente: Estimaciones propias, con base en CCP (2010 y 2015).

* Las relaciones impropias se definen conforme al criterio de edad establecido en la ley 9406 . 
En el gráfico 4, se presenta el porcentaje de relaciones impropias reportadas por la diferencia de edad con respecto a la pareja de la primera relación, con el fin de contrastar los resultados con el gráfico 2. Puede observarse que el porcentaje de relaciones impropias se incrementa proporcionalmente más para el grupo que había iniciado su vida sexual con personas de su misma edad y menos para el grupo que se inició sexualmente con personas al menos 5 años mayores. A pesar de ello, la mayor proporción de relaciones impropias se encuentran en el grupo que inició su actividad sexual con una persona al menos cinco años mayor, mientras que para el grupo que inició su vida sexual con una persona entre 2 y menos de 5 años mayor, 1 de cada 10 personas había sufrido ya, para el momento de la encuesta, una relación que constituía una forma de delito sexual. Esto podría sugerir la necesidad de contar con bases de datos longitudinales que permitan observar si la vulnerabilidad a los delitos sexuales se encuentra correlacionada a la experiencia sexual inicial.

\section{GRÁFICO 4 \\ PORCENTAJE DE PERSONAS EN LA MUESTRA BALANCEADA QUE HAN SIDO VÍCTIMAS DE RELACIONES IMPROPIAS*, SEGÚN DIFERENCIA DE EDAD RESPECTO A SU PRIMERA PAREJA}

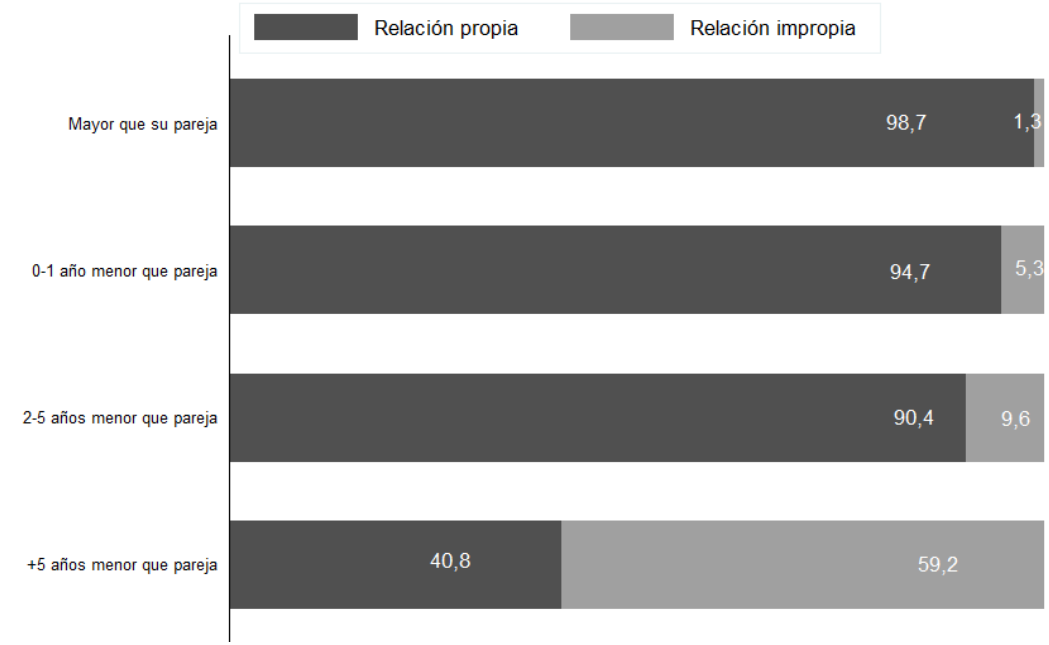

Fuente: Estimaciones propias, con base en CCP (2010 y 2015).

* Las relaciones impropias se definen conforme al criterio de edad establecido en la ley 9406 .

\section{Delito por mantener relaciones sexuales sin consentimiento}

Finalmente, $y$ con el fin de obtener una medida comprensiva sobre el nivel de los delitos sexuales existentes por mantener relaciones sexuales sin consentimiento, se procedió a agregar aquellos casos en donde la persona reportó haber sido víctima de violación. Es decir, este indicador incluye tanto las relaciones impropias (en donde una parte no tiene legalmente la facultad de consentir) como las violaciones, i.e., cuando una persona ha sido penetrada sin su consentimiento. Estas últimas son las personas que respondieron afirmativamente a la pregunta de si alguna vez habían sido forzadas físicamente a tener sexo en contra de su voluntad. Los resultados se muestran en el gráfico 5. Lo primero que debe notarse es un incremento de aproximadamente 5 p.p. en el nivel del indicador para ambos años, tal que la diferencia observada con la estimación de los delitos por relaciones impropias se mantiene para el indicador de los delitos por mantener relaciones sexuales sin consentimiento. De esta forma, el indicador asciende a 20,68\%, en la muestra de 2010 , y 24,75\%, 
en la de 2015. Además, la diferencia observada entre ambos años es significativa, lo que continúa sugiriendo un incremento en el nivel de estos delitos en los últimos años, aunque claramente se requiere de una base de datos con más observaciones temporales para poder identificar una tendencia.

En segundo lugar, se observa un incremento en los casos reportados por mujeres: mientras el delito por relaciones impropias afecta en forma similar a mujeres y hombres, los delitos de violación mediante fuerza física afectan principalmente a las mujeres. Por tanto, las mujeres pasan a ser más afectadas por estos delitos sexuales en general: ellas representan el 57,09\% del total de estos casos. En la muestra de 2010, 24\% de las mujeres fueron víctimas de relaciones sexuales no consentidas, frente a $17,29 \%$ de los hombres, siendo esta diferencia estadísticamente significativa. En la muestra de 2015, el porcentaje afectado por estos delitos sexuales se incrementa para ambos grupos y la brecha continúa siendo significativa, aunque se reduce levemente: el indicador de los delitos por mantener relaciones sexuales sin consentimiento para 2015 aumenta a 27,1\% en el caso de las mujeres y $22,3 \%$ en los hombres.

\section{GRÁFICO 5 \\ PORCENTAJE DE PERSONAS EN LA MUESTRA BALANCEADA QUE HAN SIDO VÍCTIMAS DE RELACIONES SEXUALES NO CONSENTIDAS, SEGÚN GÉNERO Y AÑO DE LA ENCUESTA}

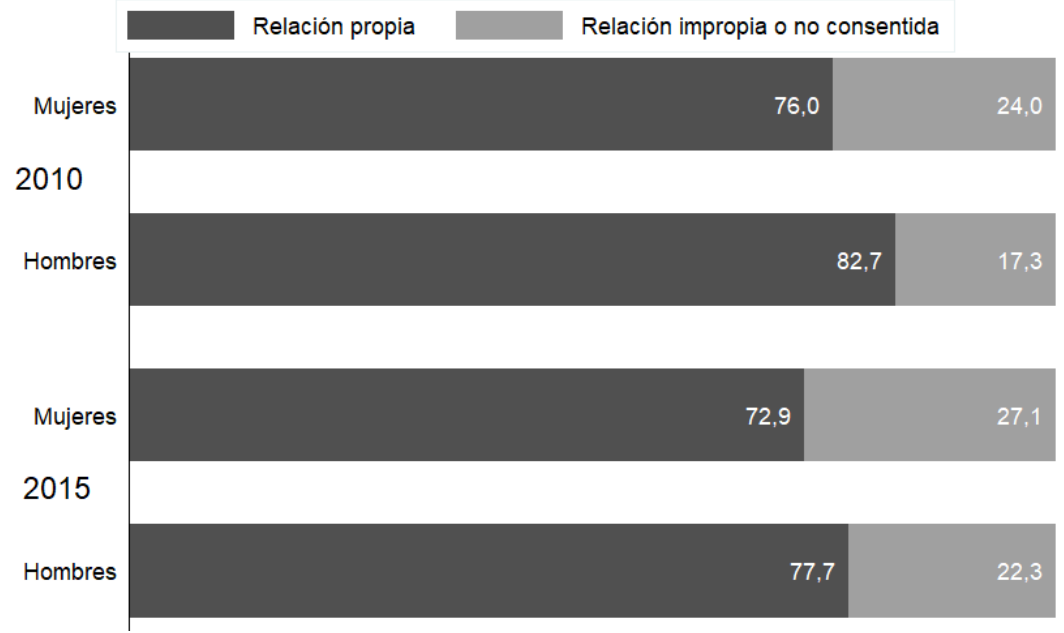

Fuente: Estimaciones propias, con base en CCP (2010 y 2015).

En tercer lugar, los datos muestran que solo 99 de las personas cuya primera experiencia sexual fue impropia reportan ser víctimas de relaciones impropias sin consentimiento (de un total de 921 casos). Esto representa apenas 10,7\% de estos casos de delitos sexuales identificados. Lo anterior sugiere que existe un alto grado de normalización de este tipo de relaciones que las hace aceptables socialmente $y$, por tanto, a la hora de preguntarle a las personas involucradas en ellas si han sido víctimas de dichos delitos sexuales, estas no se reconocen como tales. Lo anterior es consistente con los resultados encontrados por Bierie y Budd (2018), según los cuales la mayoría de personas adolescentes involucradas en encuentros sexuales perciben dichos encuentros como un acto consensuado. Lo mismo ocurre para los casos de violación por relaciones impropias en los últimos 12 meses, donde únicamente 3 de los 79 casos de abusos identificados reconocen haber sido víctimas de delitos sexuales. Esto representa una proporción aún menor (apenas 3,8\%) de los casos asociados a la primera experiencia sexual. Así, del total de casos identificados, únicamente el 30,06\% se identifica como víctima de delitos sexuales, 5,92\% coincide con casos de relaciones impropias ocurridas en los últimos 12 meses previos a la entrevista $y$, la gran mayoría, el 64,02\% 
corresponde con relaciones impropias durante la primera relación sexual. Por tanto, los delitos sexuales asociados a la falta de consentimiento durante las relaciones sexuales se encuentran fuertemente institucionalizados y no se reconocen como tales por quienes lo sufren.

\section{Edad de iniciación sexual}

Es probable que los delitos sexuales por relaciones impropias no sean reconocidos como tales por quienes lo sufren, porque la población en general se inicia sexualmente a edades cada vez más tempranas, de forma tal que los scripts asociados a la sexualidad son no solo aceptados, sino promovidos en la población menor de edad, particularmente la adolescente. El gráfico 6 muestra la edad promedio de la experiencia sexual para las diferentes cohortes por género. Puede apreciarse que la edad de iniciación sexual ha disminuido considerablemente para ambos géneros, pero la caída ha sido más abrupta para las mujeres: mientras las mujeres de las generaciones nacidas antes de 1955 se iniciaban sexualmente casi a los 20 años, las nacidas entre 1996 y 2000, lo hacían poco después de haber cumplido los 15. Además, esta tendencia a la baja es particularmente marcada para las cohortes nacidas después de 1980. Algo similar se observa en los hombres, aunque la disminución en la edad de iniciación promedio no es tan marcada, pasando de 17 a 15 años entre las cohortes nacidas antes de 1955 y la nacida entre 1996 y 2000.

\section{GRÁFICO 6 \\ EDAD PROMEDIO DE LA PRIMERA RELACIÓN SEXUAL POR COHORTE Y GÉNERO}

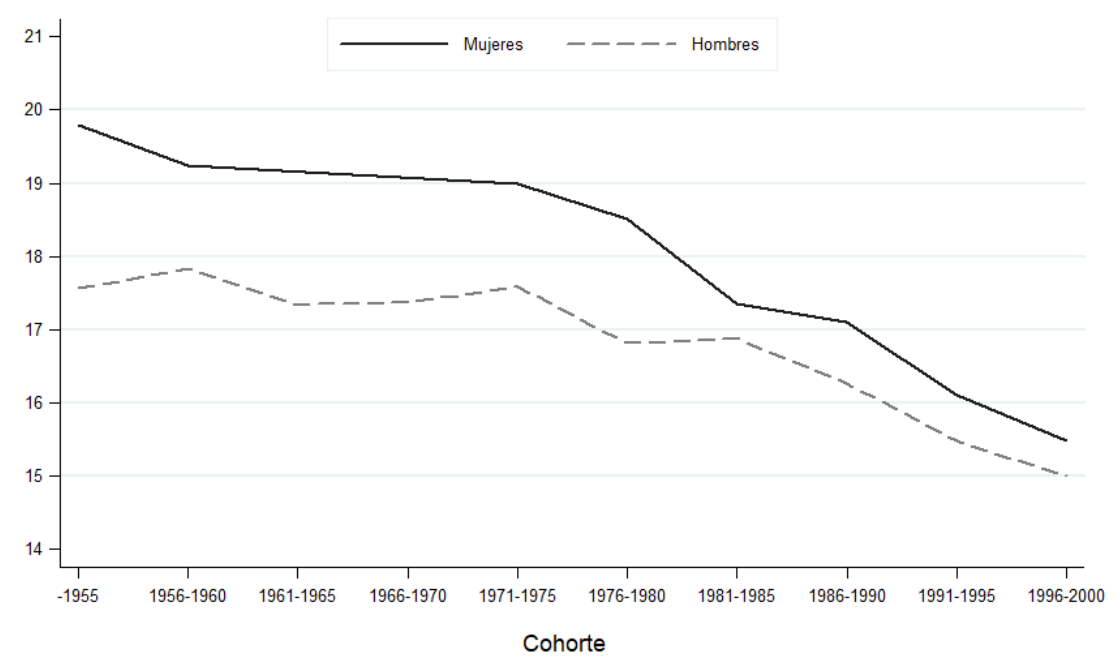

Fuente: Estimaciones propias, con base en CCP (2010 y 2015)

\section{Descripción de variables}

En esta sección, se presenta la descripción de una serie de variables que podrían estar vinculados con los delitos asociados a las relaciones sexuales no consentidas, con el fin de explorar y empezar a esbozar un perfil sobre las víctimas de ellos, en particular, aquel asociado a las relaciones impropias. Esta sección es empírica y no busca establecer relaciones causales, sino solo explorar posibles asociaciones. Los correspondientes resultados correlacionales se presentan en la sección 5. El cuadro 3 muestra las estadísticas descriptivas para la muestra balanceada. Como ya se mencionó, esta consta de 5668 observaciones. Las mujeres representan el 50,7\% de la muestra 
y las observaciones de 2010 corresponden al 51,5\% del total de observaciones. El cuadro 3 también muestra el nivel del indicador para los delitos por relaciones sexuales no consentidas.

Siguiendo a Jackson (1978), podría argumentarse que las relaciones abusivas se encuentran normalizadas dentro de los scripts de género, en tanto permiten validar la masculinidad del agresor, a la vez que pueden ser una vía para que la mujer encuentre una forma de sustento. Por ejemplo, una familia podría permitir que un hombre adulto mantenga una relación abusiva con su hija menor de edad, en tanto este la provea de bienes materiales. Esta relación podría incluso ser alentada por sus parientes si supusiesen que la menor se va a encontrar en una mejor situación económica que la que la familia le ofrece. De ser así, la familia estaría utilizando el capital erótico de su hija para procurarle un mayor bienestar económico. En circunstancias de este tipo, podría suponerse que el capital humano podría ser un sustituto del capital erótico, de forma tal que, si se le provee a la persona menor de edad de capital humano, esta luego podría ir al mercado laboral y procurarse un ingreso laboral que haría menos probable que ella sea víctima de relaciones impropias. Esto sería también consistente con el modelo de capital humano de Becker (1991), según el cual las personas con capital humano especializado en el mercado laboral no se especializarán en la producción de bienes domésticos. Por tanto, el análisis contemplará si existe algún tipo de relación entre ser víctima de estos delitos sexuales y el nivel de capital humano de la persona.

Para ello, se ha construido un indicador a partir de la clasificación utilizada por la División Estadística del Banco Central de Costa Rica para catalogar la mano de obra como no calificada cuando poseen educación primaria o menos, semi-calificada si obtuvieron un diploma de educación secundaria o técnica y calificada si obtuvieron un título universitario. Los datos muestran que casi dos tercios de las personas que respondieron la encuesta son no calificadas, lo cual es consistente con los resultados obtenidos en otras encuestas nacionales. Cerca de un cuarto de las observaciones corresponden a personas semi-calificadas y un décimo a personas calificadas. Además, los datos del 2015 muestran una mejor calificación de las mujeres, lo cual también es consistente con los resultados de otras encuestas. Ahora bien, la población no calificada se encuentra sobrerrepresentada dentro de quienes han sido víctima de relaciones sexuales no consentidas: esta representa el 77,3\% del total de casos identificados; mientras que la población semi-calificada es el 16,2\% y la calificada, el 6,5\%. En contraste, la población no calificada representa el 58,4\% de quienes no han sido víctimas de este tipo de delito; la semi-calificada, el 29,2\% y la calificada, $12,4 \%$. Así, la probabilidad de ser víctima de relaciones sexuales no consentidas para la población no calificada es 2,1 veces la de la población calificada. Para las mujeres sin calificación, en particular, la probabilidad de ser víctima de relaciones sexuales no consentidas es 2,6 veces la probabilidad de la de una mujer calificada. Lo anterior apoya la hipótesis de que el capital humano puede estar relacionado con el nivel de este tipo de delitos sexuales.

Se introduce también una variable para identificar a aquellas personas que se declaran ateas o agnósticas, con el fin de capturar algún tipo de información sobre su marco de valores y creencias. En general, una de cada diez personas en la muestra es atea o agnóstica, aunque los hombres componen casi dos tercios de este grupo. En principio, no se cuenta con ninguna premisa sobre cuál podría ser el signo esperado entre esta variable y la probabilidad de ser víctima de delitos sexuales, aunque los datos muestran que el porcentaje de los delitos sexuales aumenta para el grupo conformado por personas ateas o agnósticas (30,7\%) con respecto al del grupo creyente $(21,8 \%)$. Existen dos posibles explicaciones para esto: podría ser que las personas que han sufrido delitos sexuales tienden a perder sus creencias religiosas tras la experiencia o que, al formar parte de un grupo minoritario, esto les haga más vulnerables al ataque, pues son un grupo discriminado socialmente. 


\section{ESTADÍSTICAS DESCRIPTIVAS DE LA MUESTRA BALANCEADA}

\begin{tabular}{|c|c|c|c|c|c|c|}
\hline & \multicolumn{4}{|c|}{2010} & \multicolumn{2}{|c|}{2015} \\
\hline & Total & Mujeres & Hombres & Total & Mujeres & Hombres \\
\hline Observaciones & 2920 & 1474 & 1446 & 2748 & 1397 & 1351 \\
\hline $\begin{array}{l}\text { Porcentaje de población víctima } \\
\text { de delitos sexuales }\end{array}$ & $20,7 \%$ & $24,0 \%$ & $17,3 \%$ & $24,7 \%$ & $27,1 \%$ & $22,3 \%$ \\
\hline \multicolumn{7}{|l|}{$\begin{array}{l}\text { Distribución del nivel educativo } \\
\text { de las personas: }\end{array}$} \\
\hline No calificada & $63,8 \%$ & $64,6 \%$ & $62,9 \%$ & $61,6 \%$ & $60,3 \%$ & $62,8 \%$ \\
\hline Semi-calificada & $24,4 \%$ & $23,6 \%$ & $25,2 \%$ & $28,2 \%$ & $27,3 \%$ & $29,1 \%$ \\
\hline Calificada & $11,8 \%$ & $11,8 \%$ & $11,8 \%$ & $10,2 \%$ & $12,3 \%$ & $8,1 \%$ \\
\hline $\begin{array}{l}\text { Porcentaje de personas ateas o } \\
\text { agnósticas }\end{array}$ & $9,2 \%$ & $6,6 \%$ & $11,9 \%$ & $10,1 \%$ & $6,7 \%$ & $13,5 \%$ \\
\hline $\begin{array}{l}\text { Promedio en la escala de } \\
\text { homofobia }\end{array}$ & 0,43 & 0,40 & 0,46 & 0,35 & 0,33 & 0,37 \\
\hline \multicolumn{7}{|l|}{$\begin{array}{l}\text { Distribución de la satisfacción } \\
\text { de la comunicación con } \\
\text { progenitores antes de los } 21 \\
\text { años: }\end{array}$} \\
\hline Insatisfecha & $53,7 \%$ & $55,2 \%$ & $52,1 \%$ & $45,5 \%$ & $47,6 \%$ & $43,4 \%$ \\
\hline Poco satisfecha & $16,2 \%$ & $15,2 \%$ & $17,3 \%$ & $18,3 \%$ & $17,8 \%$ & $19,0 \%$ \\
\hline Algo satisfecha & $13,3 \%$ & $11,9 \%$ & $14,7 \%$ & $12,9 \%$ & $11,6 \%$ & $14,3 \%$ \\
\hline Muy satisfecha & $16,9 \%$ & $17,7 \%$ & $16,0 \%$ & $23,2 \%$ & $23,1 \%$ & $23,4 \%$ \\
\hline \multicolumn{7}{|l|}{$\begin{array}{l}\text { Distribución de la satisfacción } \\
\text { de la comunicación con } \\
\text { profesores antes de los } 21 \text { años: }\end{array}$} \\
\hline Insatisfecha & $51,3 \%$ & $52,0 \%$ & $50,6 \%$ & $46,8 \%$ & $48,4 \%$ & $45,2 \%$ \\
\hline Poco satisfecha & $22,8 \%$ & $22,4 \%$ & $23,3 \%$ & $23,0 \%$ & $22,3 \%$ & $23,8 \%$ \\
\hline Algo satisfecha & $15,1 \%$ & $15,0 \%$ & $15,2 \%$ & $16,6 \%$ & $16,8 \%$ & $16,4 \%$ \\
\hline Muy satisfecha & $10,8 \%$ & $10,7 \%$ & $10,9 \%$ & $13,6 \%$ & $12,6 \%$ & $14,6 \%$ \\
\hline $\begin{array}{l}\text { Porcentaje que reporta sufrir } \\
\text { acoso callejero }\end{array}$ & $87,5 \%$ & $90,2 \%$ & $84,7 \%$ & $84,2 \%$ & $90,3 \%$ & $77,8 \%$ \\
\hline Porcentaje que es mujer & $50,5 \%$ & $100,0 \%$ & $0,0 \%$ & $50,8 \%$ & $100,0 \%$ & $0,0 \%$ \\
\hline Edad promedio (en años) & 35,4 & 35,6 & 35,1 & 34,9 & 35,8 & 34,0 \\
\hline Porcentaje que es migrante & $8,4 \%$ & $8,3 \%$ & $8,4 \%$ & $8,2 \%$ & $8,9 \%$ & $7,4 \%$ \\
\hline \multicolumn{7}{|l|}{$\begin{array}{l}\text { Distribución según región } \\
\text { donde habita: }\end{array}$} \\
\hline Central & $65,3 \%$ & $65,0 \%$ & $65,6 \%$ & $64,2 \%$ & $65,8 \%$ & $62,6 \%$ \\
\hline Chorotega & $7,5 \%$ & $8,1 \%$ & $6,9 \%$ & $8,1 \%$ & $8,0 \%$ & $8,3 \%$ \\
\hline Pacífico Central & $5,6 \%$ & $5,6 \%$ & $5,6 \%$ & $5,3 \%$ & $3,9 \%$ & $6,7 \%$ \\
\hline Brunca & $7,0 \%$ & $7,2 \%$ & $6,8 \%$ & $6,7 \%$ & $6,1 \%$ & $7,3 \%$ \\
\hline Huetar Atlántica & $10,0 \%$ & $9,8 \%$ & $10,2 \%$ & $8,9 \%$ & $8,5 \%$ & $9,3 \%$ \\
\hline Huetar Norte & $4,7 \%$ & $4,4 \%$ & $5,0 \%$ & $6,8 \%$ & $7,7 \%$ & $5,9 \%$ \\
\hline
\end{tabular}

Fuente: Estimaciones propias, con base en CCP (2010 y 2015). 
Se creó una escala de homofobia como instrumento para medir el marco referencial de los individuos. Este es un índice de 0 a 1 creado a partir del promedio del puntaje de diez afirmaciones evaluadas en una escala Likert de cuatro puntos, donde 1 implica el nivel más alto de homofobia. Las encuestas le solicitan a las personas identificar en una escala Likert de cuatro puntos su nivel de acuerdo con las siguientes afirmaciones: "me incomoda que me vean en compañía de una persona homosexual", "una persona homosexual no debe trabajar con niños y niñas", "las personas homosexuales tienden a ser sexualmente promiscuas", "es chocante observar a una pareja homosexual besándose", "las personas homosexuales siempre tienen modales típicos del sexo opuesto", "las personas homosexuales no deben frecuentar los mismos lugares públicos que el resto", "una persona homosexual tiene el derecho de poder desempeñar cualquier trabajo que escoja", "puedo ser amigo o amiga de una persona homosexual", "las parejas homosexuales deben tener derecho a adoptar niños y niñas" y "debería permitirse la unión civil entre personas homosexuales". Las últimas cuatro afirmaciones se codificaron en forma inversa para obtener el promedio del puntaje. En la muestra, los hombres obtienen un puntaje promedio superior al de las mujeres, indicando que presentan mayores niveles de homofobia, aunque esta diferencia es menor para la encuesta de 2015.

Se incluyeron otras dos variables como instrumentos para capturar el nivel de confianza que la persona tenía en su juventud a las personas que la rodeaban en su cotidianidad. Estas son dos variables relacionadas con la satisfacción de la comunicación con sus progenitores y sus profesores antes de los veintiún años. La hipótesis es que una persona que se siente segura con las figuras de autoridad estará más protegida y recibirá mejor información sobre cómo resguardarse de relaciones sexuales no consentidas. Los datos muestran que la mayoría de las personas, indistintamente de su género, reportan haberse sentido insatisfechas con el nivel de comunicación con sus progenitores en la encuesta de 2010. Prácticamente, dos terceras partes de las personas encuestadas entonces registran un nivel de insatisfacción o poca satisfacción. Además, la distribución es bastante similar entre mujeres y hombres, por lo que podría suponerse que las personas emplean las mismas estrategias de comunicación con sus hijas e hijos. En la encuesta de 2015, el indicador mejora levemente, aunque la mayoría de las personas continúan estando insatisfechas o poco satisfechas con la comunicación con sus progenitores. El indicador para medir el nivel de satisfacción con sus profesores es aún peor que el observado con sus progenitores. Aquí, en ambas encuestas, el nivel de insatisfacción o poca satisfacción agrupa al menos al 70\% del total de personas encuestadas. Esto sugiere que las relaciones en la escuela y colegio son percibidas como menos cercanas y seguras que aquellas que se entablan en el seno familiar.

Se incorpora también a la muestra una variable dicotómica que toma el valor de 1 cuando la persona reporta sufrir acoso callejero. Con esto se pretende capturar información sobre el contexto social en el que se desenvuelve la persona. La premisa es que personas en contextos donde se perciben situaciones de violencia generalizada, tal como el acoso callejero, se desenvuelven en ambientes de mayor violencia donde la agresión sexual podría estar más normalizada. Los datos apoyan esta premisa, pues el porcentaje de víctimas de relaciones sexuales no consentidas es mayor para el grupo que reporta acoso callejero (23,2\%) que el observado para el grupo que no sufre de dicho acoso (19,3\%). Además, los datos muestran que la mayoría de las personas sufren este tipo de violencia callejera, aunque las mujeres reportan mayores niveles de afectación que los hombres, de $90 \%$ en ambas encuestas. Sin embargo, los hombres también reportan tasas de acoso elevadas y superiores al $75 \%$.

Finalmente, se introducen cuatro variables de control sociodemográfico: dos variables dicotómicas para identificar a las mujeres y migrantes, una variable categórica para la región de residencia y una variable continua para la edad de la persona. Como ya se mencionó antes, las mujeres conforman la mitad de la muestra, pero representan el 57\% de los casos de relaciones sexuales no consentidas identificados. Esta diferencia radica, principalmente, en que las mujeres 
sufren más casos de violación. Por otra parte, la muestra cuenta con una población migrante algo superior al 8\% en ambos años; casi dos terceras partes de la muestra residen en la región central del país y la edad promedio de las personas encuestadas ronda los 35 años. Todas estas variables son similares entre los géneros y los diferentes años.

\subsection{Método}

Se estimó cuatro modelos de regresión logit con el fin de determinar si ser víctima de delitos sexuales se asocia con las variables relacionadas con capital humano, la identidad, el contexto en el que se desarrolla la persona y otras características sociodemográficas. En cada caso, la probabilidad de ser víctima de delitos sexuales $\left(y_{i}=1\right)$ se deriva del siguiente modelo:

$$
\operatorname{Pr}\left(y_{i} \neq 0 \mid x_{i}\right)=\frac{e^{x_{i} \gamma}}{1+e^{x_{i} \gamma}}
$$

donde $x$ es la matriz de características individuales y $\gamma$ es el vector de coeficientes. Las variables incluidas en $x$ varían en cada modelo. En el modelo A, se regresó ser víctima de delitos sexuales sobre la variable de capital humano, las dos variables sobre identidad, i.e., que la persona sea atea o agnóstica y el índice de homofobia, las tres variables sobre el contexto relacionadas con el nivel de satisfacción con progenitores y profesores en la juventud y si sufren acoso callejero actualmente $y$, finalmente, la variable dicotómica para identificar a las mujeres. Como esta última es significativa, los resultados se presentan desagregados para mujeres y hombres. En el modelo B se incluyen como variables de control sociodemográfico la edad y su cuadrado, ser migrante, la región de residencia y una variable dicotómica para identificar la encuesta de 2015. Los modelos C y D presentan los resultados de la regresión para las muestras de los años 2010 y 2015 en forma separada, debido a que efectivamente existen diferencias entre ambas encuestas. Estos fueron utilizando el comando logit de Stata 12. Debido a que los resultados obtenidos de un modelo logit no pueden interpretarse directamente, se reportan los efectos marginales medios para todas las covariables, con el objetivo de facilitar la interpretación de los resultados. Estos corresponden a los cambios (derivadas) en las respuestas ante un cambio en la covariable y se obtienen mediante el comando "margins" de postestimación en Stata.

\section{RESULTADOS}

El cuadro 4 presenta los efectos marginales medios para los resultados de la regresión logit? Debe recordarse que estos resultados muestran la existencia de relaciones correlacionales, mas no así causales entre las variables, de manera que informan sobre el perfil asociada a estas víctimas de relaciones sexuales no consentidas. El modelo A muestra que, efectivamente, el capital humano se encuentra asociado negativa y significativamente con la probabilidad de ser víctima de relaciones sexuales no consentidas, a los niveles de significancia estándar. Es, además, la variable que muestra una mayor asociación: tener un grado de semi-calificación le reduce a las mujeres la probabilidad de ser víctima de estos delitos sexuales en 14,2 p.p. y en 11 p.p. a los hombres con respecto a una persona sin calificaciones; mientras que ser calificada reduce dicha probabilidad en 19,9 y 8,6 p.p. a las mujeres y los hombres, respectivamente. Debe notarse, además, que el efecto de la educación en la reducción de la probabilidad de sufrir relaciones sexuales no consentidas en las mujeres es creciente.

7 En el anexo puede consultarse los resultados de la regresión, pero para facilitar la interpretación en esta sección se analizarán los efectos marginales medios. 
Por su parte, que la persona sea atea o agnóstica se relaciona positivamente con haber sufrido relaciones sexuales no consentidas, en particular, para las mujeres. Como ya se mencionó anteriormente, esto podría deberse a que, al ser parte de un grupo minoritario, las personas no creyentes sean más vulnerables a estos delitos. Tal y como lo menciona Jackson (1978), el abuso puede servir como un instrumento para castigar a quienes no se conforman con los patrones o scripts tradicionales; de forma que abusar de o agredir sexualmente a una persona no creyente en una sociedad religiosa podría bien fungir como instrumento de coerción. También podría ser que, al ser víctimas de relaciones sexuales no consentidas, las personas se planteen y sobrelleven un proceso de cuestionamiento más profundo que las conduce a escapar de las religiones y a asumir formas de vida más racionales y menos míticas. La otra variable asociada a la identidad, la puntuación en la escala de homofobia y que reflejaba el nivel de conservadurismo de la persona, no resultó significativa en ninguno de los casos.

Las variables asociadas al nivel de satisfacción con la comunicación con sus progenitores $y$ profesores antes de los veintiún años resultan significativas. Para la muestra agregada, quienes reportan poca satisfacción con la comunicación con sus progenitores ven reducirse la probabilidad de ser víctima de relaciones sexuales no consentidas en 4,6 p.p., en promedio, respecto a quienes reportaron insatisfacción, mientras que la probabilidad se reduce en 6,9 p.p. para quienes reportaron alguna o mucha satisfacción. El efecto en estas dos últimas categorías es, además, superior para las mujeres que los hombres. Similarmente, reportar alguna satisfacción con la comunicación con profesores reduce en al menos 5 p.p. la probabilidad de ser víctima de estos delitos sexuales, aunque esta variable no pareciera ser robusta para el caso de los hombres. Lo anterior sugiere que sentir seguridad en el ambiente familiar probablemente es un factor más robusto para brindarle protección a las personas menores de edad que el contexto escolar, lo cual, además, pareciera lógico dado que el hogar representa el espacio de mayor intimidad.

En contraste, si la persona reporta sufrir acoso callejero, su probabilidad de haber sido víctima de relaciones sexuales no consentidas es mayor en todos los casos, a un nivel de confianza de $90 \%$. Para las mujeres, esta probabilidad se incrementa en 10,8 p.p. y, para los hombres, en 3,7 p.p. Dado que esta relación no es causal, no se puede saber si las personas que han sufrido relaciones sexuales no consentidas son más sensibles y efectivas en detectar y reconocer el acoso callejero o si estas personas viven en un contexto social de mayor violencia y, por tanto, están más expuestas a todo tipo de agresión. Finalmente, ser mujer se asocia con un incremento de 5,3 p.p. en la probabilidad de ser víctima de relaciones sexuales no consentidas, lo que apoya el argumento de que las mujeres son más vulnerables al abuso o a la agresión sexual.

La introducción de las variables de control sociodemográficas en el modelo B no cambia significativamente los resultados encontrados en el modelo A. Ni la edad, la edad al cuadrado ni ser migrante resultan significativas. En cuanto a las regiones, los datos sugieren que la probabilidad de ser víctima de relaciones sexuales no consentidas se incrementa para los hombres en el Pacífico Central y para ambos géneros en la región Huetar Atlántica. De hecho, en estas regiones el porcentaje de la población víctima de estos delitos sexuales supera el 23\% que se observa, en promedio, para todas las regiones. En la región Pacífico Central este indicador alcanza el valor de 29,5\%, mientras que es 31,4\% en la región Huetar Atlántica. La primera de estas regiones está conformada por los cantones de San Mateo y Orotina en la provincia de Alajuela y los cantones de Esparza, Aguirre, Parrita, Garabito, Montes de Oro y Puntarenas, en la provincia de Puntarenas, por lo que cabría la pena explorar si este mayor riesgo de ser víctima de relaciones sexuales no consentidas para los hombres en dicha región podría estar asociado con mayores niveles de prostitución masculina y tráfico de drogas en algunos de estos cantones. Por su parte, la región Huetar Atlántica está conformada por los cantones de Limón, Pococí, Siquirres, Talamanca, Matina y Guácimo en la provincia de Limón, lo que podría señalar la existencia de valores culturales más permisivos respecto a estos delitos sexuales en esta región en comparación con la región Central. 
Por último, los datos de la encuesta de 2015 son significativamente diferentes a los del 2010, por lo que los modelos $\mathrm{C}$ y D presentan los resultados de la regresión en forma desagregada para ambas muestras.

En general, los resultados obtenidos para el modelo B se mantienen cuando se analizan únicamente los datos de la encuesta de 2010 (modelo C) para la muestra agregada y las mujeres, con dos excepciones: en primer lugar, se pierde la significancia del coeficiente asociado a reportar poca satisfacción con la comunicación con profesores. Esto sugiere que, para lograr disminuir la incidencia de este tipo de delitos sexuales, mantener un grado de comunicación que genera insatisfacción o poca satisfacción en la persona menor de edad es insuficiente y, por tanto, se debe promover que la persona efectivamente se sienta satisfecha con esta comunicación para poder asegurar un ambiente de protección. En segundo lugar, se pierde el grado de significancia del coeficiente asociado a la región Huetar Atlántica para las mujeres. De hecho, la incidencia de los delitos sexuales reportados en esta región para los hombres es de 31,3\%, 2 puntos porcentuales superior a la incidencia registrada para las mujeres. En el modelo masculino, se pierde la significancia para la mayoría de las variables, de forma que sufrir relaciones sexuales no consentidas únicamente está vinculado con ser ateo o agnóstico y con vivir en la región Pacífico Central o Huetar Atlántica. Lo anterior señala la necesidad de estudiar más a fondo este tema en los hombres.

Los resultados en el modelo D, referidos a la muestra de 2015, son consistentes con los del modelo B para la muestra agregada. En el caso de las mujeres, los resultados son robustos tanto con los del modelo B como el modelo $\mathrm{C}$, excepto porque se pierde la significancia del coeficiente asociado a tener mucha satisfacción con la comunicación con profesores y el coeficiente asociado a la región Huetar Atlántica vuelve a ser significativo a los niveles de confianza estándar. En el modelo masculino, el capital humano y la satisfacción con la comunicación con los profesores están negativa y significativamente asociados a la probabilidad de ser víctima de relaciones sexuales no consentidas. En contraste, sufrir de acoso callejero y vivir en el Pacífico Central se relacionan positivamente con la probabilidad de ser víctima de relaciones sexuales no consentidas. 


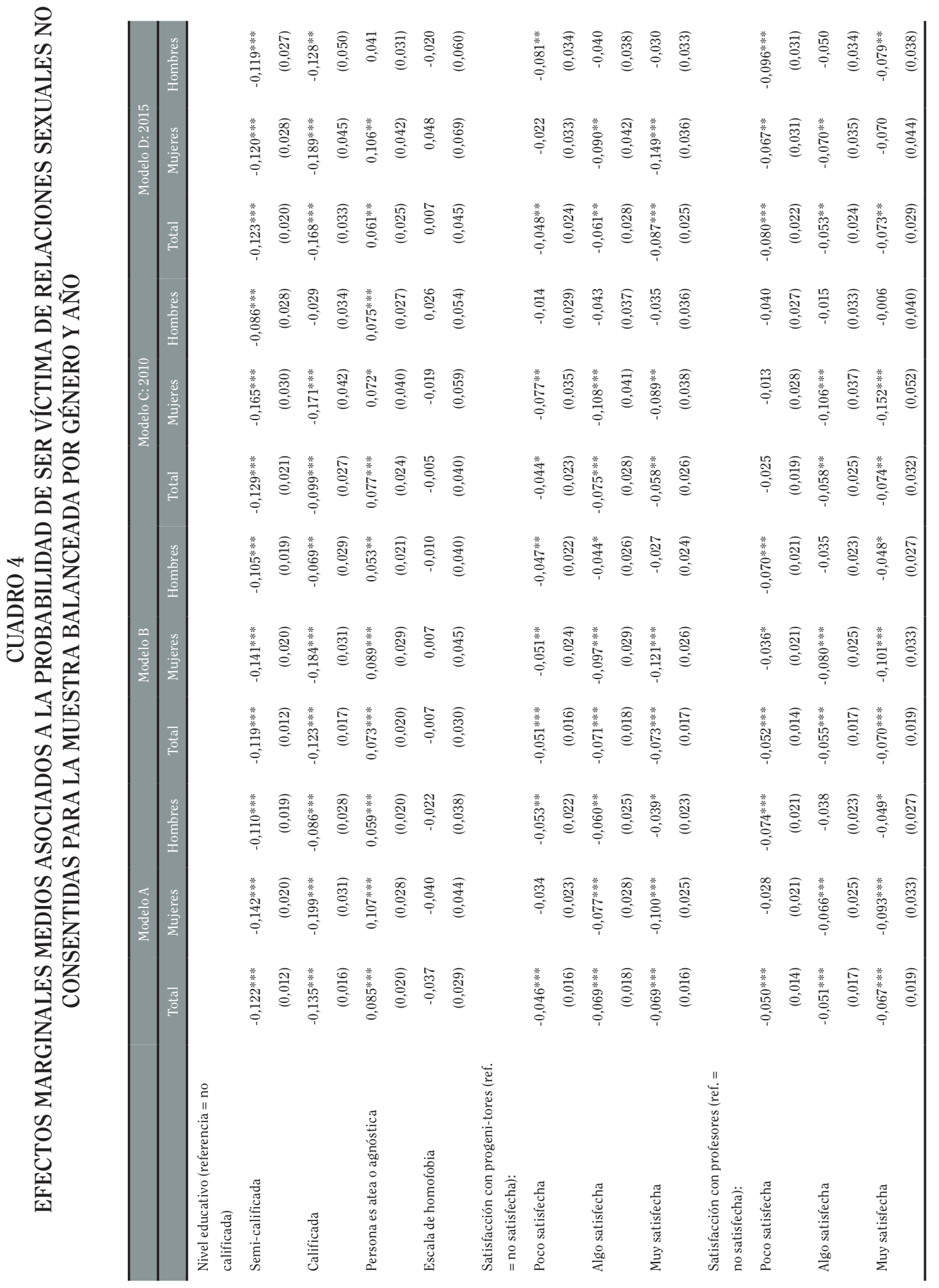




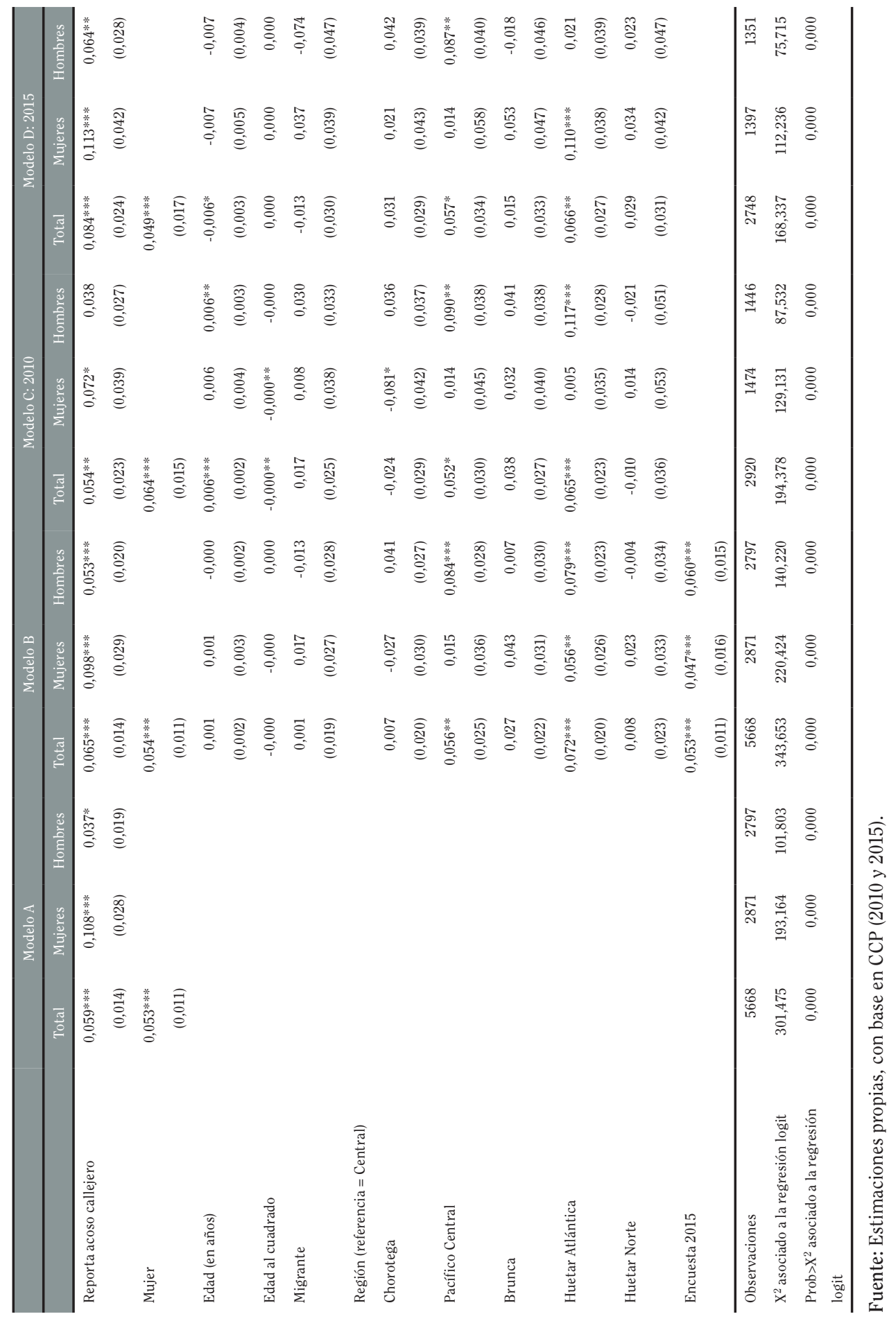




\section{CONCLUSIONES}

Suponiendo las definiciones dadas por la ley 9406, la encuesta solo ha permitido hacer una estimación conservadora sobre el nivel de los delitos asociados a las relaciones sexuales no consentidas, pero hemos llegado a cuatro conclusiones importantes del análisis de los datos: (i) al menos una quinta parte de la población mayor de 15 años en Costa Rica ha sido víctima de este tipo de delitos sexuales. (ii) Las relaciones impropias con personas menores de edad afectan a mujeres y hombres en forma similar, aunque los delitos sexuales utilizando formas de violencia más explícitas afectan más a las mujeres que a los hombres. (iii) La tasa de los delitos sexuales asociados a las relaciones sexuales no consentidas estimada es, además, estadísticamente superior en la muestra de 2015 que la de 2010, lo que sugiere un incremento en el nivel de violencia sexual experimentado por la población. Y (iv) la mayor parte de estos delitos no es reconocida como tal por la población. De hecho, únicamente el $10 \%$ de las personas que han sufrido relaciones sexuales no consentidas en la muestra reportan haber sido víctimas de estos delitos sexuales. Esto último ciertamente apunta a una normalización de los delitos sexuales en la sociedad costarricense y a la necesidad de tener una discusión más amplia que permita entender la aceptación cultural de este tipo de relaciones.

Aun así, en segundo lugar, las personas no suelen conocer la ley, ni en su primera relación sexual parece haber conocimiento de lo que la relación significa, ni el hecho que mediara de alguna manera presión o manipulación. Pese a que la historia de las figuras jurídicas muestra el interés de una sociedad por regular tales o cuales conductas, la "visibilización legal" de dichos comportamientos no conduce necesariamente a la "percepción y comprensión" de lo que significan, por parte de las víctimas, en particular cuando estas son menores de edad. Entonces, en este caso, el consentimiento no puede emerger ni siquiera como cuestión, porque no entiende la víctima qué consiente o porque legalmente se dice que no puede consentir. De esta manera, la representación de una relación consentida (la primera relación) pasa significativamente por ignorar las garantías legales (no saber que se trata de una relación impropia) y por ignorar cuáles son las condiciones en que se encuentra el individuo, en general.

En tercer lugar, a la vez la ley tanto configura la relación impropia como establece las condiciones de vulnerabilidad a las que se somete el sujeto. Al estatuir rango de edad y diferencias de edad con la pareja, supone precisamente que dentro de esos rangos y diferencias el individuo tiene una madurez sexual y que no es vulnerable al abuso, violencia o agresión, y por el contrario, se constituye en delito sexual cuando está fuera de ese rango y esas diferencias. Ciertamente, el problema de la vulnerabilidad consiste en que sea legal y desde cierto punto de vista nominal, pues substrae al individuo decidir sobre sí mismo. La ley protege, en principio, la integridad y la libertad sexual de las personas y lo hace a través del rango y diferencias de edad, que substituyen el criterio del individuo en su defensa. La encuesta revela el grado de vulnerabilidad de las personas en su primera relación sexual, y la reforma legal podría subrayar dicha vulnerabilidad. 


\section{REFERENCIAS}

Alfonso X el Sabio (2006). Las siete partidas. Biblioteca Virtual Universal (Trad.). https://biblioteca. org.ar/libro.php?texto=130949

Asamblea Legislativa (15 de noviembre de 1970). Ley 4573 Código Penal. La Gaceta, 257. http:// www.pgrweb.go.cr/scij/Busqueda/Normativa/Normas/nrm_norma.aspx?param1=NRM\&nValo r1=1\&nValor2=5027\&nValor3=5321\&strTipM=FN

Asamblea Legislativa (13 de enero de 2017). Ley 9406 Fortalecimiento de la protección legal de las niñas y las adolescentes mujeres ante situaciones de violencia de género asociadas a relaciones abusivas, reforma Código Penal, Código Familia, Ley Orgánica TSE y Registro Civil, y Código Civil. La Gaceta, 10.

Bierie, D. M., \& Budd, K. M. (2018). Romeo, Juliet, and Statutory Rape. Sexual Abuse, 30(3), 296321. https://doi.org/10.1177/1079063216658451

Bullough, V. L. (2005). Age of consent. Journal of Psychology \& Human Sexuality, 16(2-3), 25-42. http://dx.doi.org/10.1300/J056v16n02_03.

Centro Centroamericano de Población (2010) Encuesta Nacional de Salud Sexual y Reproductiva. Universidad de Costa Rica.

Centro Centroamericano de Población (2015) Encuesta Nacional de Salud Sexual y Reproductiva. Universidad de Costa Rica. https://ccp.ucr.ac.cr/index.php/farmacoeconomia-pareo/pareoprincipal/240-ii-encuesta-nacional-de-salud-sexual-y-salud-reproductiva.html

Cocca, C. (2015) Age of consent. In P. Whelehan \& A. Bolin (Ed.) The International Encyclopedia of Human Sexuality. John Wiley \& Sons, Inc. http://dx.doi.org/10.1002/9781118896877

Congreso de la República de Costa Rica (30 de agosto de 1941). Código Penal. La Gaceta, 192. http:// www.pgrweb.go.cr/scij/Busqueda/Normativa/Normas/nrm_norma.aspx?param1=NRM\&nValo r1=1\&nValor2=37382\&nValor3=39414\&strTipM=FN

Consejo Nacional de la República de Costa Rica (27 de abril de 1880). Código Penal de la República de Costa Rica.

http://www.pgrweb.go.cr/scij/Busqueda/Normativa/Normas/nrm_texto_completo.aspx?param1=NR TC\&nValor1=1\&nValor2=7306\&nValor3=7824\&strTipM=TC

Edward I (1275). The Statute of Westminster. https://ucadia.s3.amazonaws.com/acts_ uk/1200_1299/uk_act_1275_statute_westminster.pdf

Ehrlich, J. S. (2006). From age of consent laws to the "Silver Ring Thing": the regulation of adolescent female sexuality. Health Matrix: The Journal of Law-Medicine, 16(1), 151-181. https://scholarlycommons.law.case.edu/healthmatrix/voll6/iss1/8/

Ellis, S. J., \& Kitzinger, C. (2002). Denying equality: An analysis of arguments against lowering the age of consent for sex between men. Journal of Community \& Applied Social Psychology, 12(3), 167-180. https://doi.org/10.1002/casp.670

Estado de Costarrica (30 de julio de 1841). Código General del Estado de Costarrica. Imprenta del Estado.

Frank, D. J., Camp, B. J. \& Boutcher, S. A. (2010). Worldwide trends in the criminal regulation of sex, 1945 to 2005. American Sociological Review, 75(6), 867-893. https://doi. org/10.1177/0003122410388493

Fraisse, G. (2017). Du consentement (éd. augmentée). Seuil. 
Horvath, M. A. H. \& Giner-Sorolla, R. (2007). Below the age of consent: influences on moral and legal judgments of adult-adolescent sexual relationships. Journal of Applied Social Psychology, 37(12), 2980-3009. https://doi.org/10.1111/j.1559-1816.2007.00290.x

Jackson, S. (1978). The social context of rape: sexual scripts and motivation. Women's Studies International Quarterly, I, 27-38. https://doi.org/10.1016/S0148-0685(78)90311-1

Jackson, S. \& Scott, S. (2015). A sociological history of researching childhood and sexuality: continuities and discontinuities. In E. Renold, J. Ringrose \& D. Egan (Eds.). Children, sexuality and sexualization. Palgrave Macmillan, (pp. 39-55).

Janssen, D. F. (2015). Anthropologizing young/minor sexualities. In E. Renold, J. Ringrose, \& D. Egan (Eds.). Children, sexuality and sexualization. Palgrave Macmillan.

Murigi, E. (2018). Age of consent around the world. https://www.worldatlas.com/articles/age-ofconsent-around-the-world.html

Office of the High Commissioner, United Nations Human Rights [OHCHR] (1989). Convention on the Rights of the Child. https://www.ohchr.org/en/professionalinterest/pages/crc.aspx

Presidente Constitucional de la República (30 de noviembre de 1918). Código Penal de Costa Rica de 1918.

http://www.pgrweb.go.cr/scij/Busqueda/Normativa/Normas/nrm_norma.aspx?param1=NRM\&nValo r1=1\&nValor2=77202\&nValor3=96630\&strTipM=FN

Presidente Constitucional de la República de Costa Rica (22 de abril de 1924). Código Penal de 1924. http://www.pgrweb.go.cr/scij/Busqueda/Normativa/Normas/nrm_norma.aspx?param1=NRM \&nValor1=1\&nValor2=35220\&nValor3=37141\&strTipM=FN

Sutherland, K. (2003). From jailbird to jailbait: age of consent law and the construction of teenage sexualities. William \& Mary Journal of Women and the Law, 9(3), 313-349. http://scholarship. law.wm.edu/wmjowl/vol9/iss3/2

UNICEF (2018). 20 years of the Convention on the Rights of the Child. Are you old enough...to have sex?. https://www.unicef.org/rightsite/433_457.htm

Waites, M. (2003). Equality at last? Homosexuality, heterosexuality and the age of consent in the United Kingdom. Sociology, 37(4), 637-655. https://doi.org/10.1177/00380385030374001

Este artículo se encuentra disponible mediante la licencia Creative Commons Reconocimiento-NoComercial-SinObraDerivada 3.0 Costa Rica. Para mayor información escribir a revista.iice@ucr.ac.cr. 


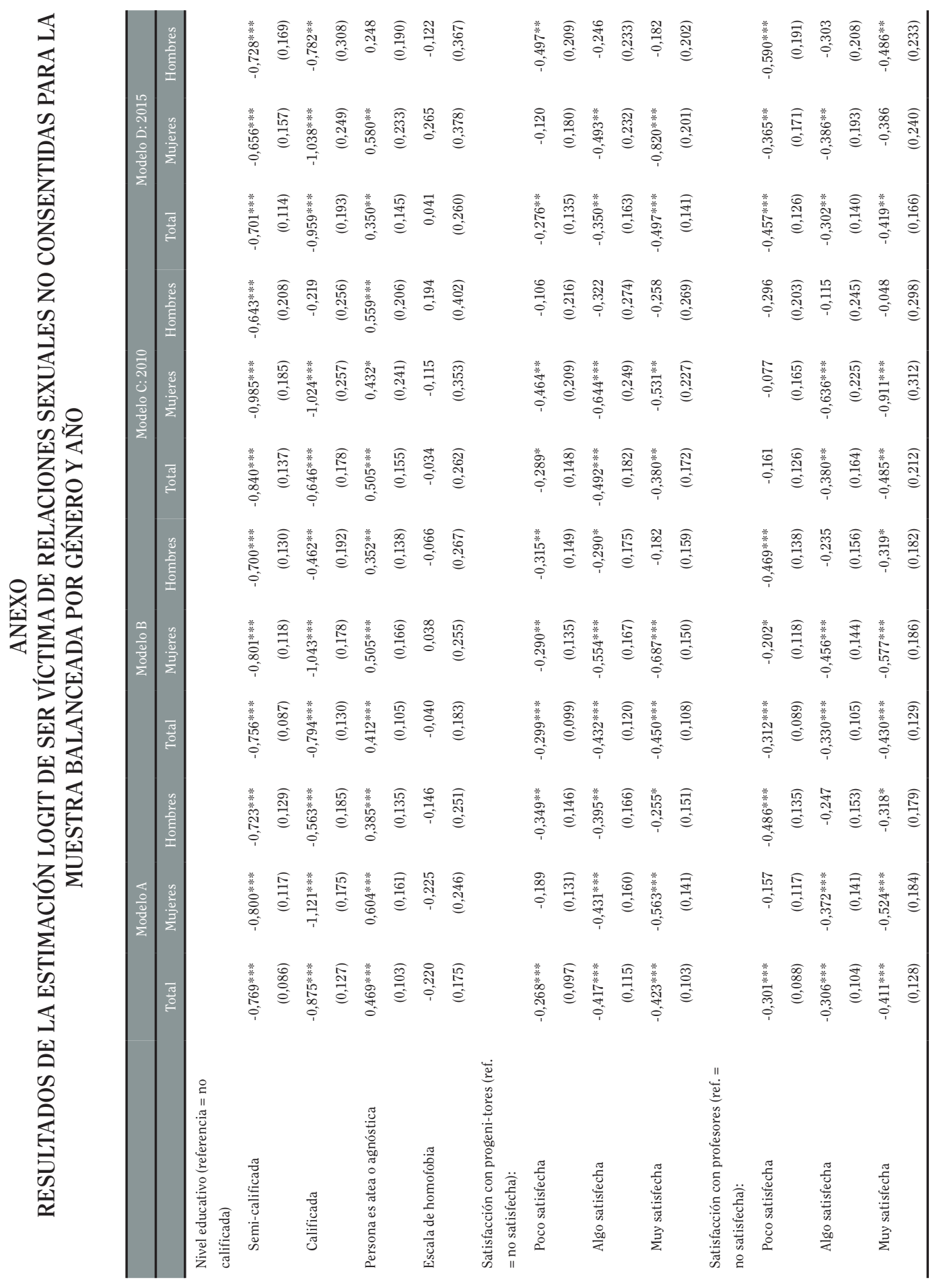




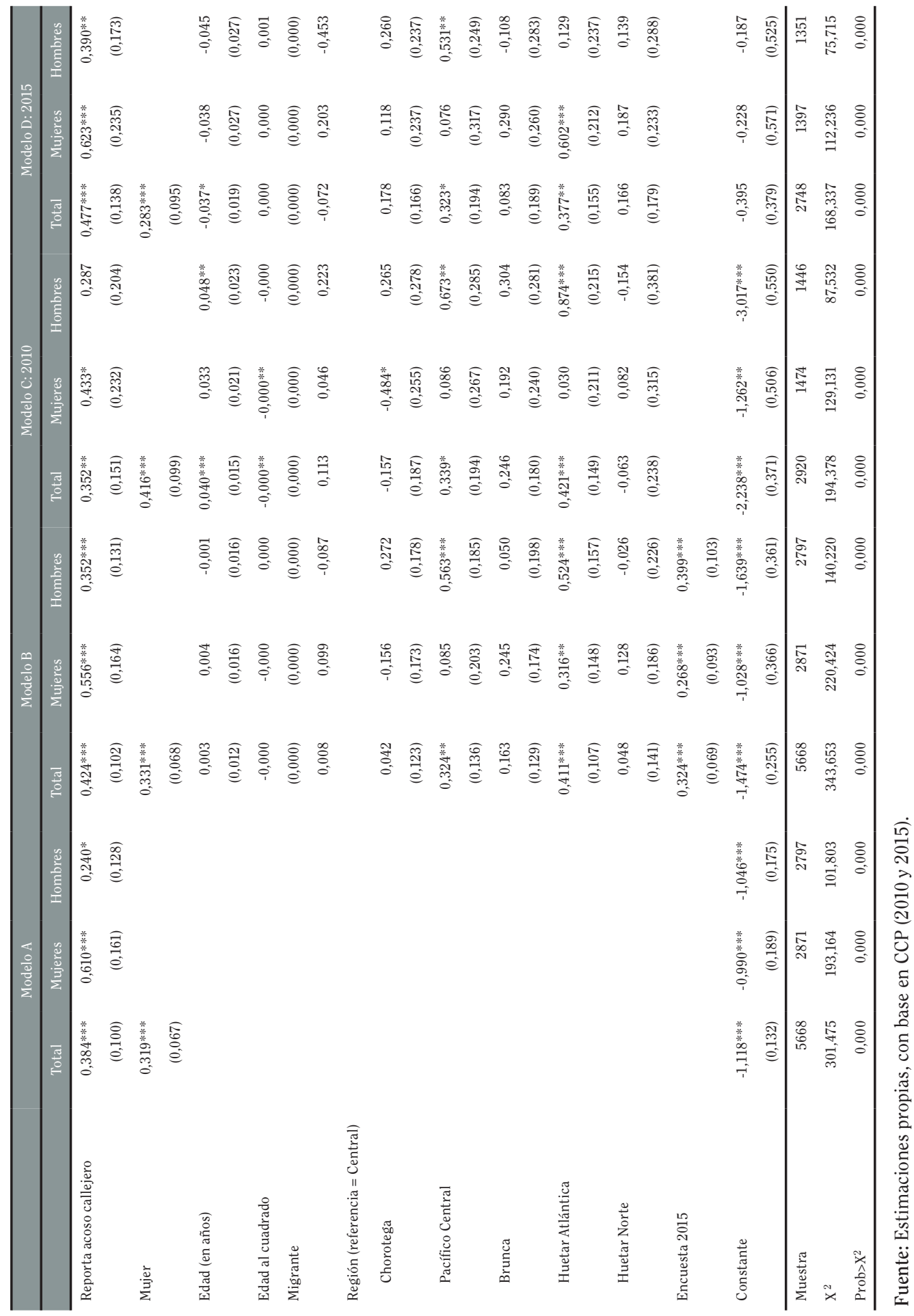

